\title{
Analysis of linear reactive general rate model of liquid chromatography considering irreversible and reversible reactions
}

\author{
Seemab Bashir ${ }^{\mathrm{a}}$, Shamsul Qamar ${ }^{\mathrm{a}, \mathrm{b}, *}$, Sadia Perveen ${ }^{\mathrm{a}}$, Andreas Seidel-Morgenstern ${ }^{\mathrm{b}}$ \\ ${ }^{a}$ Department of Mathematics, COMSATS Institute of Information Technology, \\ Park Road Chak Shahzad Islamabad, Pakistan \\ ${ }^{b}$ Max Planck Institute for Dynamics of Complex Technical Systems, \\ Sandtorstrasse 1, 39106 Magdeburg, Germany
}

\begin{abstract}
A linear general rate model of two-component liquid chromatography is analyzed considering heterogenous reactions of types $\mathrm{A} \rightarrow \mathrm{B}$ and $\mathrm{A} \rightleftarrows \mathrm{B}$. The model equations incorporate axial dispersion, external and intra particle pore diffusions, interfacial mass transfer, linear sorption kinetics, and first order heterogeneous chemical reactions. The solution methodology successively employs the Laplace transform and linear transformation steps to uncouple the governing set of coupled differential equations. The resulting system of uncoupled ODEs is solved by applying an elementary solution technique. The numerical Laplace inversion is employed to transform back the solutions in the actual time domain. The current solutions extend and generalize the recent solutions of nonreactive general rate model for single-solute transport. For validation, a high resolution finite volume scheme is implemented to obtain the numerical solutions. Different case studies are considered to verify the correctness of semi-analytical solutions and the accuracy of the numerical scheme. To further study the behavior of a chromatographic reactor, numerical temporal moments of the elution profiles are presented for both reactant and product. The derived semi-analytical solutions are useful tools to study the influence of solid phase reaction rate constant, interfacial mass transfer rate, intra particle pore diffusion, and reactant adsorption affinity on the concentration profiles.
\end{abstract}

\footnotetext{
${ }^{*}$ Corresponding author. Tel: +49-391-6110454; fax: +49-391-6110500

Email addresses: qamar@mpi-magdeburg.mpg.de (Shamsul Qamar)
} 
Key words: Chromatographic reactor, linear general rate model, irreversible and reversible reactions, linear adsorption, finite volume scheme, moment analysis.

\section{Introduction}

High performance liquid chromatography is considered as an efficient quantitative and qualitative chromatographic tool in chemical engineering for the separation of multi component mixtures in which each component has different adsorption affinity. Chromatographic techniques have numerous advantages and play key roles in chemical, pharmaceutical, petrochemical, bio-technical, photochemical and food industries. There are several characteristics which make the HPLC procedures more superior over other forms of chromatography namely, (i) it is universally applicable and only few samples are excluded from the possibility of HPLC, (ii) its analysis time is short, (iii) its operation on much larger scale is possible, (iv) it has remarkable assay precision $( \pm 0.5 \%)$, and (v) HPLC columns are reusable without regeneration [1-3].

Reactive chromatography is an integrated process in which chemical or biochemical reactions are combined with the chromatographic separation [1-4]. This technique improves the conversion of reactants and purity of products and has, therefore, attracted several researchers in the past few decades [2, 4-26].

The chemical reactions inside this integrated process can be catalyzed homogeneously and heterogeneously. In the case of homogeneous catalysis, the separation of catalyst has to be taken into account. On the other hand, heterogeneously catalyzed reactions usually occur in the case of esterification, where the same ion exchange resin act as a catalyst for the reaction and as an absorbent for the separation. To understand the basic principle of a fixed-bed chromatographic reactor, let us consider a single column reactor and a reversible reaction of the type $\mathrm{A} \rightleftarrows \mathrm{B}$. The reactant $A$ is dissolved in the desorbent and is injected as rectangular pulse into the column packed with the stationary phase. The reaction occurs at the surface of catalyst to form the product $B$. Both components $A$ and $B$ interact with the surface of adsorbent and because of their different affinities to the stationary phase, they move inside the column with different propagation speeds. Hence, components are 
separated and the driving force for the forward reaction is enhanced and the backward reaction is suppressed. As a result, chemical equilibrium can be achieved and high purity product can be obtained at the column outlet.

Mathematical modeling and simulation of chromatography are useful tools to understand the involved transport mechanisms, to scale up physio-chemical parameters, and to optimize the experimental conditions. Several models exist in the literature with different levels of complexities to describe the process $[2,3]$. The general rate model (GRM) is the most complicated and detailed model in chromatography among all the transport models [3]. In GRM, chromatographic separation is governed by several sorption and transport mechanisms at different scales. Molecules in a sample of mixture are transported by convection through interstitial bulk phase between the chromatographic beads and are dispersed due to inhomogeneities in the flow. The external film mass transfer resistances equilibrate the concentration gradients between the bulk phase and the stagnant film round the porous beads. The intra particle diffusion (combination of pore and surface diffusion), difference in the adsorption/desorption rate and equilibria make possible the separation of individuals components from complex mixtures.

The analytical solutions of linear chromatographic models can be used to quantify the effect to different mass transfer and reaction kinetics on the process without doing practical experiments in the laboratories $[3,5,19,20,27-36]$. These models are useful to understand the chromatographic process, as in most of the situations the injected volume of the sample is small and is diluted. The derived analytical solutions could be used to validate the numerical solutions of more complex models when no experimental data is available.

The Laplace domain solutions can be used to derive the statistical temporal moments [3, 34-36]. The retention equilibrium-constant and parameters of the mass transfer kinetics in the column are related to the moments. The moment analysis has been used in a number of studies of fixed-bed systems [3, 34-38].

In this work, a linear reactive general rate model (RGM) is analyzed to study twocomponent adsorption equilibria and reaction-separation kinetics in a fixed-bed chromatographic reactor. Semi-analytical solutions of the model are derived for both irreversible 
and reversible reactions in the particles macropores. The Laplace transformation and eigen-decomposition technique are successively applied to solve the model equations for two different sets of boundary conditions (BCs), namely the Dirichlet and the Danckwerts BCs. Due to the complex structure of the solutions, analytical back transformations are not possible. Therefore, numerical Laplace inversion is applied to get the time domain solutions $[19,20,34,35]$. To validate the derived solutions, numerical solutions are also obtained by applying a high resolution finite volume scheme to the same model equations [39-41]. Several case studies are considered and both numerical and semi-analytical results are compared. The behavior of chromatographic reactor is further analyzed by calculating analytical and numerical moments of concentration profile.

The major novelty of this article specifically include: (a) derivation of semi-analytical solutions of a linear two-component reactive GRM for two different sets of boundary conditions, (b) consideration solid-phase reactions, (c) numerical inversion of solutions from frequency to time domain, (d) calculation of numerical moments, and (e) validation of the correctness of analytical results by a successful comparison with numerical results. The considered two-component reactive GRM contains four coupled PDEs, two PDEs describe concentration balances in the bulk of fluid and two PDEs are concentration balances in the particles pores. The analytical solutions of this model for the considered reaction types and boundary conditions are more complicated than the classical single-component non-reactive GRM [35]. Besides the Laplace transformation, a successive application of decoupling technique is required to uncouple the resulting coupled system of ODEs obtained after applying Laplace transformation. Especially, in the reversible reaction case, this technique is applied two times which is a more uphill task. Thus, the analysis and the results presented below are truly new and nontrivial. The analytical Laplace inversion is not possible due to very complicates expressions of the Laplace domain solutions. Although analytical Laplace inversion is not possible, the derived semi-analytical solutions are still very accurate and useful. These solutions can be used to study the chromatographic behaviors, such as peak area, sample retention time, band broadening, asymmetry of elution profiles, and efficiency of the column. The derived semi-analytical solutions are seen as 
helpful for further developments of chromatographic reactors. For instance, the analysis could be used to study the effects of mass transfer and reaction kinetics on the elution profiles, for sensitivity analysis, for validating numerical solutions, and for estimating longitudinal dispersion coefficients from experimentally determined elution profiles, among others.

This article is organized as follows. In Section 2, the linear RGRM is introduced for irreversible reaction along with two sets of BCs, such as Dirichlet and Danckwerts BCs. In Section 3, analytical solutions are derived to solve these model equations for the considered irreversible reaction. In Section 4, this analytical solution methodology is extended to the case of reversible reaction. Section 5 introduces moment analysis. In Section 6, several test problems are considered. Conclusions are drawn in Section 7.

\section{Irreversible reaction $(A \rightarrow B)$ in a fixed-bed chromatographic reactor}

In this process, the component $A$ (component 1) converts to component $B$ (component 2) through an irreversible first order heterogeneous reaction. The reactant and product travel along the column by axial dispersion and conversion of reactant into the product is due to the first order chemical reaction in the solid phase. The considered two-component reactive general rate model (RGRM) is based upon the following assumptions:

1. The chromatographic process is isothermal.

2. The porous particles in the column are spherical shaped and have same diameter.

3. The concentration gradient in the radial direction are neglected.

4. There exist an instantaneous local equilibrium between the macropore surfaces and the stagnant fluid inside particles macropores.

5. Interfacial mass transfer between the bulk fluid and particle phases is described by film mass transfer mechanism.

6. The diffusional and mass transfer parameters are constant and are independent of the mixing effects of the component involved.

Based on the above assumption, the current RGRM contains four mass balance equations 
for the two-component mixture transport, i.e. two equations are describing transport in the bulk of fluid and two equations are for transport inside the particles macropores.

The mass balance equations of RGRM for the bulk of fluid considering two-component solute percolating through a chromatographic reactor filled with spherical particles of radius $R_{p}$ are expressed as $[2,3,35]$

$$
\begin{aligned}
& \frac{\partial c_{1}}{\partial t}+u \frac{\partial c_{1}}{\partial z}=D_{z} \frac{\partial^{2} c_{1}}{\partial z^{2}}-\frac{3}{R_{p}} F k_{\mathrm{ext}, i}\left(c_{1}-\left.c_{p, 1}\right|_{r=R_{p}}\right) \\
& \frac{\partial c_{2}}{\partial t}+u \frac{\partial c_{2}}{\partial z}=D_{z} \frac{\partial^{2} c_{2}}{\partial z^{2}}-\frac{3}{R_{p}} F k_{\mathrm{ext}, i}\left(c_{2}-\left.c_{p, 2}\right|_{r=R_{p}}\right)
\end{aligned}
$$

In the above equation, $t$ denotes the time coordinate, $z$ represents the axial coordinate along the column length, $c_{i}(t, z)$ is the concentration of $i$-th component in the bulk of fluid and $c_{p, i}(t, z)$ is the $i$-th component concentration in the particles, respectively. Moreover, $u$ is the interstitial velocity, $D_{z}$ represents the axial dispersion coefficient, $k_{\text {ext }, i}$ is the external mass transfer coefficient of $i$-th component, $F=\frac{1-\epsilon}{\epsilon}$, is the phase ratio, $\epsilon \in(0,1)$ is the external porosity and $r$ denotes the radial coordinate of spherical particles of radius $R_{p}$. The mass balance equations for the solute considering irreversible reactions in the stationary phase can be expressed assuming two mechanisms of intraparticle transport $[2,3,35]$ :

$$
\begin{aligned}
\epsilon_{p} \frac{\partial c_{p, 1}}{\partial t}+\left(1-\epsilon_{p}\right) \frac{\partial q_{p, 1}^{*}}{\partial t} & =\frac{1}{r^{2}} \frac{\partial}{\partial r}\left(r^{2}\left[\epsilon_{p} D_{p, 1} \frac{\partial c_{p, 1}}{\partial r}+\left(1-\epsilon_{p}\right) D_{s, 1} \frac{\partial q_{p, 1}^{*}}{\partial r}\right]\right)-\left(1-\epsilon_{p}\right) \nu_{1} q_{p, 1}^{*} \\
\epsilon_{p} \frac{\partial c_{p, 2}}{\partial t}+\left(1-\epsilon_{p}\right) \frac{\partial q_{p, 2}^{*}}{\partial t} & =\frac{1}{r^{2}} \frac{\partial}{\partial r}\left(r^{2}\left[\epsilon_{p} D_{p, 2} \frac{\partial c_{p, 2}}{\partial r}+\left(1-\epsilon_{p}\right) D_{s, 2} \frac{\partial q_{p, 2}^{*}}{\partial r}\right]\right)+\left(1-\epsilon_{p}\right) \nu_{1} q_{p, 1}^{*} .
\end{aligned}
$$

Here, $q_{i}^{*}$ is the local equilibrium concentration of solute in the stationary phase, $\epsilon_{p}$ is the internal porosity, $D_{p, i}$ is the pore diffusivity of $i$-th component, $D_{s, i}$ is the surface diffusivity, and $\nu_{1}$ is the reaction rate constant of component 1 .

Eqs. (1)-(4) are connected at $r=R_{p}$ via the following expressions which quantify the 
temporal change of the average loadings of the particles $[3,35]$ :

$$
\begin{aligned}
& {\left[\epsilon_{p} D_{p, 1} \frac{\partial c_{p, 1}}{\partial r}+\left(1-\epsilon_{p}\right) D_{s, 1} \frac{\partial q_{p, 1}^{*}}{\partial r}\right]_{r=R_{p}}=k_{\mathrm{ext}, 1}\left(c_{1}-\left.c_{p, 1}\right|_{r=R_{p}}\right),} \\
& {\left[\epsilon_{p} D_{p, 2} \frac{\partial c_{p, 2}}{\partial r}+\left(1-\epsilon_{p}\right) D_{s, 2} \frac{\partial q_{p, 2}^{*}}{\partial r}\right]_{r=R_{p}}=k_{\mathrm{ext}, 2}\left(c_{2}-\left.c_{p, 2}\right|_{r=R_{p}}\right) .}
\end{aligned}
$$

Only linear adsorption isotherms are considered in this work [3]:

$$
q_{p, 1}^{*}=a_{1} c_{p, 1}, \quad q_{p, 2}^{*}=a_{2} c_{p, 2}
$$

After using Eq. (7) in Eqs. (3) and (4), we obtain

$$
\begin{aligned}
& a_{1}^{*} \frac{\partial c_{p, 1}}{\partial t}=\frac{D_{\mathrm{eff}, 1}}{r^{2}} \frac{\partial}{\partial r}\left(r^{2} \frac{\partial c_{p, 1}}{\partial r}\right)-\left(1-\epsilon_{p}\right) \nu_{1} a_{1} c_{p, 1}, \\
& a_{2}^{*} \frac{\partial c_{p, 2}}{\partial t}=\frac{D_{\mathrm{eff}, 2}}{r^{2}} \frac{\partial}{\partial r}\left(r^{2} \frac{\partial c_{p, 2}}{\partial r}\right)+\left(1-\epsilon_{p}\right) \nu_{1} a_{1} \nu c_{p, 1},
\end{aligned}
$$

with,

$$
a_{i}^{*}=\epsilon_{p}+\left(1-\epsilon_{p}\right) a_{i} \text { and } D_{\mathrm{eff}, \mathrm{i}}=\epsilon_{p} D_{p, i}+\left(1-\epsilon_{p}\right) D_{s, i} a_{i}, \quad i=1,2 .
$$

Similarly, Eq. (5) and Eq. (6) simplifies to

$$
\begin{aligned}
& \left.D_{\mathrm{eff}, 1} \frac{\partial c_{p, 1}}{\partial r}\right|_{r=R_{p}}=k_{\mathrm{ext}, 1}\left(c_{1}-\left.c_{p, 1}\right|_{r=R_{p}}\right), \\
& \left.D_{\mathrm{eff}, 2} \frac{\partial c_{p, 2}}{\partial r}\right|_{r=R_{p}}=k_{\mathrm{ext}, 2}\left(c_{2}-\left.c_{p, 2}\right|_{r=R_{p}}\right) .
\end{aligned}
$$

To further simplify the analysis and reduce the number of variables, the following dimensionless variables are introduced:

$$
\begin{aligned}
& x=\frac{z}{L}, \quad \tau=\frac{u t}{L}, \quad \rho=\frac{r}{R_{p}}, \quad P e_{l}=\frac{L u}{D_{z}}, \quad \omega_{i}=\frac{L}{u} \nu_{i} a_{i}, \\
& B i_{p, i}=\frac{k_{\mathrm{ext}, \mathrm{i}} R_{p}}{D_{\mathrm{eff}, i}}, \quad \eta_{p, i}=\frac{D_{\mathrm{eff}, i} L}{R_{p}^{2} u}, \quad \xi_{p, i}=3 B i_{p, i} \eta_{p, i} F, \quad i=1,2,
\end{aligned}
$$

where $L$ is the length of the column and $P e_{l}$ is the Pectlet number. Using the above 
dimensionless variables in Eqs. (1), (2), (8) and (9), we obtain

$$
\begin{aligned}
\frac{\partial c_{1}}{\partial \tau}+\frac{\partial c_{1}}{\partial x} & =\frac{1}{P e_{l}} \frac{\partial^{2} c_{1}}{\partial x^{2}}-\xi_{p, 1}\left(c_{1}-\left.c_{p, 1}\right|_{\rho=1}\right), \\
\frac{\partial c_{2}}{\partial \tau}+\frac{\partial c_{2}}{\partial x} & =\frac{1}{P e_{l}} \frac{\partial^{2} c_{1}}{\partial x^{2}}-\xi_{p, 2}\left(c_{2}-\left.c_{p, 2}\right|_{\rho=1}\right), \\
a_{1}^{*} \frac{\partial c_{p, 1}}{\partial \tau} & =\eta_{p, 1} \frac{1}{\rho^{2}} \frac{\partial}{\partial \rho}\left(\rho^{2} \frac{\partial c_{p, 1}}{\partial \rho}\right)-\left(1-\epsilon_{p}\right) \omega_{1} c_{p, 1}, \\
a_{2}^{*} \frac{\partial c_{p, 2}}{\partial \tau} & =\eta_{p, 2} \frac{1}{\rho^{2}} \frac{\partial}{\partial \rho}\left(\rho^{2} \frac{\partial c_{p, 2}}{\partial \rho}\right)+\left(1-\epsilon_{p}\right) \omega_{1} c_{p, 1} .
\end{aligned}
$$

Eqs. (16) and (17) can now be rephrased as

$$
\begin{aligned}
& a_{1}^{*} \frac{\partial}{\partial \tau}\left[\rho c_{p, 1}\right]-\eta_{p, 1} \frac{\partial^{2}}{\partial \rho^{2}}\left[\rho c_{p, 1}\right]+\left(1-\epsilon_{p}\right) \omega_{1}\left[\rho c_{p, 1}\right]=0 \\
& a_{2}^{*} \frac{\partial}{\partial \tau}\left[\rho c_{p, 2}\right]-\eta_{p, 2} \frac{\partial^{2}}{\partial \rho^{2}}\left[\rho c_{p, 2}\right]-\left(1-\epsilon_{p}\right) \omega_{1}\left[\rho c_{p, 2}\right]=0 .
\end{aligned}
$$

Moreover, appropriate inlet and outlet BCs are needed for Eqs. (14), (15), (18) and (19). For an initially regenerated column, the corresponding initial conditions of Eqs. (14) and (15) are given as

$$
c_{i}(0, x)=0, \quad(0<x<1) \quad i=1,2
$$

and initial conditions for Eqs. (18) and (19), considering empty particles, are given as

$$
q_{p, i}^{*}(0, x, \rho)=0, \quad c_{p, i}(0, x, \rho)=0, \quad x, r \in(0,1)
$$

Due to the assumed rapid adsorption or desorption rates, the concentrations of solute in the pores and that in the stationary phase are in equilibrium state.

In this study, the following two sets of BCs are considered for Eqs. (14) and (15).

\section{Type 1: Dirichlet inlet BCs}

In this case, the simpler Dirichlet BCs are applied at the column inlet:

$$
c_{i}(\tau, x=0)=\left\{\begin{array}{lc}
c_{\mathrm{inj}, i}, & \text { if } \quad 0 \leq \tau \leq \tau_{\mathrm{inj}}, \\
0, & \tau>\tau_{\mathrm{inj}},
\end{array}\right.
$$

together with zero Neumann BCs for a hypothetically infinite length column:

$$
\left.\frac{\partial c_{i}}{\partial x}\right|_{x=\infty}=0
$$


In the above equation, $\tau_{\text {inj }}$ denotes the time of sample injection. For sufficiently small dispersion coefficient, for example $D_{z} \leq 10^{-5} \mathrm{~m}^{2} / \mathrm{s}$, the Dirichlet inlet boundary conditions are well applicable.

\section{Type 2: Danckwerts inlet BCs}

In this case, the Robin type BCs, also known as Danckwerts BCs in chemical engineering, are applied at the column inlet [42]:

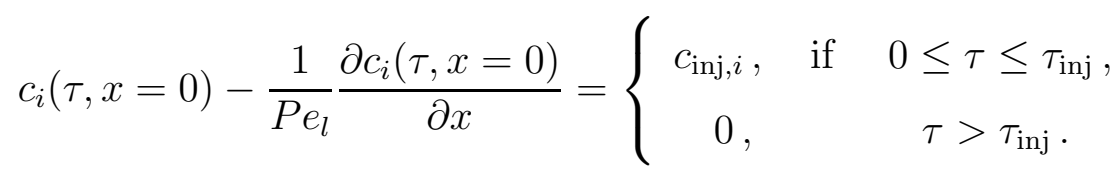

At the outlet of the column of finite length $x=1$, the following Neumann BCs are used

$$
\left.\frac{\partial c_{i}}{\partial x}\right|_{x=1}=0
$$

The natural boundary condition at $\rho=0$ and $\rho=1$ are assumed for Eqs. (18) and (19) (c.f. Eqs. (11) and (12))

$$
\left.\frac{\partial c_{p_{i}}}{\partial \rho}\right|_{\rho=0}=0,\left.\quad \frac{\partial c_{p_{i}}}{\partial \rho}\right|_{\rho=1}=B i_{p, i}\left(c_{i}-\left.c_{p_{i}}\right|_{\rho=1}\right) .
$$

\section{Analytical solutions of RGRM for reaction of type $A \rightarrow B$}

In this section, semi-analytical solutions of linear RGRM (c.f. Eqs. (14), (15), (18) and (19)) are derived for Dirichlet (Eqs. (22a) and (22b)) and Danckwert (Eqs. (23a) and (23b)) boundary conditions. The model can be conveniently solved by means of Laplace transformation which is defined as

$$
\bar{c}(s, x)=\int_{0}^{\infty} \mathrm{e}^{-\mathrm{s} \tau} \mathrm{c}(\tau, \mathrm{x}) \mathrm{d} \tau, \quad \tau \geq 0 .
$$

The Laplace transformations of model Eqs. (14) and (15) yield

$$
\begin{aligned}
& s \overline{c_{1}}+\frac{d \overline{c_{1}}}{d x}=\frac{1}{P e_{l}} \frac{d^{2} \overline{c_{1}}}{d x^{2}}-\xi_{p, 1}\left(\overline{c_{1}}-\left.\bar{c}_{p, 1}\right|_{\rho=1}\right), \\
& s \overline{c_{2}}+\frac{d \overline{c_{2}}}{d x}=\frac{1}{P e_{l}} \frac{d^{2} \overline{c_{2}}}{d x^{2}}-\xi_{p, 2}\left(\overline{c_{2}}-\left.\bar{c}_{p, 2}\right|_{\rho=1}\right) .
\end{aligned}
$$


While, the Laplace transformations of Eqs. (18) and (19) give

$$
\begin{aligned}
& \frac{d^{2}}{d \rho^{2}}\left[\rho \bar{c}_{p, 1}\right]-\frac{a_{1}^{*} s}{\eta_{p, 1}}\left[\rho \bar{c}_{p, 1}\right]-\frac{\left(1-\epsilon_{p}\right) \omega_{1}}{\eta_{p, 1}}\left[\rho \bar{c}_{p, 1}\right]=0, \\
& \frac{d^{2}}{d \rho^{2}}\left[\rho \bar{c}_{p, 2}\right]-\frac{a_{2}^{*} s}{\eta_{p, 2}}\left[\rho \bar{c}_{p, 2}\right]+\frac{\left(1-\epsilon_{p}\right) \omega_{1}}{\eta_{p, 2}}\left[\rho \bar{c}_{p, 1}\right]=0 .
\end{aligned}
$$

The general solution of Eq. (28) is given as

$$
\bar{c}_{p, 1}(s, x, \rho)=\frac{1}{\rho}\left[k_{1} e^{\sqrt{\alpha(s)} \rho}+k_{2} e^{-\sqrt{\alpha(s)} \rho}\right],
$$

where,

$$
\alpha(s)=\frac{a_{1}^{*} s+\left(1-\epsilon_{p}\right) \omega_{1}}{\eta_{p, 1}} .
$$

On utilizing the boundary conditions given in Eq. (24), the values of $k_{1}$ and $k_{2}$ in Eq. (30) come out to be

$$
k_{1,2}= \pm \frac{B i_{p, 1} \overline{c_{1}}}{2 \sinh (\sqrt{\alpha(s)})\left[\sqrt{\alpha(s)} \operatorname{coth}(\sqrt{\alpha(s)})+B i_{p, 1}-1\right]} .
$$

Here, the upper positive sign is taken for $k_{1}$ and the lower negative sign for $k_{2}$. At $\rho=1$, Eqs. (30) and (32) reduce to

$$
\left.\bar{c}_{p, 1}\right|_{\rho=1}=\bar{c}_{1} f_{1}(s)
$$

where

$$
f_{1}(s)=\frac{B i_{p, 1}}{\left[\sqrt{\alpha(s)} \operatorname{coth}(\sqrt{\alpha(s)})+B i_{p, 1}-1\right]} .
$$

After introducing Eq. (33) in Eq. (29), we obtain the general solution as

$$
\bar{c}_{p, 2}(s, x, \rho)=\frac{1}{\rho}\left[k_{1}^{\prime} e^{\sqrt{\alpha^{\prime}(s)} \rho}+k_{2}^{\prime} e^{-\sqrt{\alpha^{\prime}(s)} \rho}\right]+\frac{f_{1}(s)\left(1-\epsilon_{p}\right) \omega_{1} \overline{c_{1}}}{a_{2}^{*} s} .
$$

where $\alpha^{\prime}(s)=\frac{a_{2}^{*} s}{\eta_{p, 2}}$. By using Eq. (24) in Eq. (35), we obtian

$$
k_{1,2}^{\prime}= \pm \frac{B i_{p, 2} \overline{c_{2}}-\frac{B i_{p, 2} f_{1}(s)\left(1-\epsilon_{p}\right) \omega_{1} \bar{c}_{1}}{a_{2}^{*} s}}{2 \sinh \left(\sqrt{\alpha^{\prime}(s)}\right)\left[\sqrt{\alpha^{\prime}(s)} \operatorname{coth}\left(\sqrt{\alpha^{\prime}(s)}\right)+B i_{p, 2}-1\right]},
$$


At $\rho=1$, Eqs. (35) and (36) reduce to

$$
\left.\bar{c}_{p, 2}\right|_{\rho=1}=\overline{c_{2}} f_{2}(s)+\overline{c_{1}} A(s)
$$

where,

$$
f_{2}(s)=\frac{B i_{p, 2}}{\left[\sqrt{\alpha^{\prime}(s)} \operatorname{coth}\left(\sqrt{\alpha^{\prime}(s)}\right)+B i_{p, 2}-1\right]}
$$

and

$$
A(s)=\frac{f_{1}(s)\left(1-\epsilon_{p}\right) \omega_{1}}{a_{2}^{*} s}\left[\frac{-B i_{p, 2}}{\left[\sqrt{\alpha^{\prime}(s)} \operatorname{coth}\left(\sqrt{\alpha^{\prime}(s)}\right)+B i_{p, 2}-1\right]}+1\right] .
$$

After introducing Eqs. (33) and (37) in Eqs. (26) and (27), respectively, we get the following ordinary differential equations (ODEs)

$$
\begin{aligned}
& \frac{d^{2} \overline{c_{1}}}{d x^{2}}-P e_{l} \frac{d \overline{c_{1}}}{d x}-P e_{l} \phi_{1}(s) \overline{c_{1}}=0 . \\
& \frac{d^{2} \overline{c_{2}}}{d x^{2}}-P e_{l} \frac{d \overline{c_{2}}}{d x}-P e_{l} \frac{d \overline{c_{2}}}{d x}=P e_{l} \xi_{p, 2} A(s) \overline{c_{1}},
\end{aligned}
$$

where

$$
\phi_{1}(s)=s+\xi_{p, 1}\left(1-f_{1}(s)\right), \quad \phi_{2}(s)=s+\xi_{p, 2}\left(1-f_{2}(s)\right) .
$$

In the matrix notions, Eqs. (40) and (41) can be expressed as

$$
\frac{d^{2}}{d x^{2}}\left(\begin{array}{l}
\bar{c}_{1} \\
\bar{c}_{2}
\end{array}\right)-P e_{l} \frac{d}{d x}\left(\begin{array}{c}
\bar{c}_{1} \\
\bar{c}_{2}
\end{array}\right)-\left[\begin{array}{cc}
P e_{l} \phi_{1}(s) & 0 \\
-\xi_{p, 2} P e_{l} A(s) & P e_{l} \phi_{2}(s)
\end{array}\right]\left(\begin{array}{l}
\bar{c}_{1} \\
\bar{c}_{2}
\end{array}\right)=\left(\begin{array}{l}
0 \\
0
\end{array}\right) .
$$

Here, the square brackets [ ] stands for a square matrix, the curly brackets ( ) represents a column vector, and $\bar{c}_{i}$ for $(i=1,2)$ are the liquid phase concentrations of mixture components in the Laplace domain.

The reaction coefficient matrix $[B]$ on in Eq. (43) is given as

$$
B=\left[\begin{array}{cc}
P e_{l} \phi_{1}(s) & 0 \\
-\xi_{p, 2} P e_{l} A(s) & P e_{l} \phi_{2}(s)
\end{array}\right]
$$


In the next step, linear transformation matrix $[A]$ will be computed $[19,20,43]$. Note that, the columns of $[A]$ are the eigenvectors of the matrix $[B]$. The eigenvalues and eigenvectors of the matrix $[B]$ are given as:

$$
\lambda_{1}=P e_{l} \phi_{1}(s), \quad x_{1}=\left(\begin{array}{c}
A_{11} \\
\frac{-\xi_{p, 2} P e_{l} A(s) A_{11}}{P e_{l} \phi_{1}(s)-P e_{l} \phi_{2}(s)}
\end{array}\right) \quad \text { and } \quad \lambda_{2}=P e_{l} \phi_{2}(s), \quad x_{2}=\left(\begin{array}{c}
0 \\
A_{22}
\end{array}\right) \text {. }
$$

Here, $\lambda_{1}$ and $\lambda_{2}$ denote the eigenvalues and $A_{11}$ and $A_{22}$ are the arbitrary constants. For simplicity, we take the values of $A_{11}$ and $A_{22}$ equal to one. Using Eq. (45), the diagonal matrix $\kappa$ and the transformation matrix $[A]$ can be written as

$$
\kappa=\left[\begin{array}{cc}
P e_{l} \phi_{1}(s) & 0 \\
0 & P e_{l} \phi_{2}(s)
\end{array}\right], \quad A=\left[\begin{array}{cc}
1 & 0 \\
\frac{-\xi_{p, 2} P e_{l} A(s)}{P e_{l} \phi 1(s)-P e_{l} \phi_{2}(s)} & 1
\end{array}\right] .
$$

The matrix $[A]$ is then used in the following linear transformation $[20,43]$

$$
\left(\begin{array}{l}
\bar{c}_{1} \\
\bar{c}_{2}
\end{array}\right)=\left[\begin{array}{cc}
1 & 0 \\
\frac{-\xi_{p, 2} P e_{l} A(s)}{P e_{l} \phi_{1}(s)-P e_{l} \phi_{2}(s)} & 1
\end{array}\right]\left(\begin{array}{l}
b_{1} \\
b_{2}
\end{array}\right)
$$

Applying the above linear transformation on Eq. (43), we get

$$
\frac{d^{2}}{d x^{2}}\left(\begin{array}{l}
b_{1} \\
b_{2}
\end{array}\right)-P e_{l} \frac{d}{d x}\left(\begin{array}{l}
b_{1} \\
b_{2}
\end{array}\right)=\left[\begin{array}{cc}
P e_{l} \phi_{1}(s) & 0 \\
0 & P e_{l} \phi_{2}(s)
\end{array}\right]\left(\begin{array}{l}
b_{1} \\
b_{2}
\end{array}\right)
$$

Eq. (48) represents a system of two independent ODEs. Their explicit solutions are given as

$$
b_{1}(s, x)=A_{1} e^{m_{1} x}+B_{1} e^{m_{2} x}, \quad m_{1,2}=\frac{P e_{l}}{2}\left(1 \mp \sqrt{1+\frac{4 \phi_{1}(s)}{P e_{l}}}\right)
$$

and

$$
b_{2}(s, x)=A_{2} e^{m_{3} x}+B_{2} e^{m_{4} x}, \quad m_{3,4}=\frac{P e_{l}}{2}\left(1 \mp \sqrt{1+\frac{4 \phi_{2}(s)}{P e_{l}}}\right) .
$$

Here, $A_{1}, B_{1}, A_{2}$ and $B_{2}$ are constants of integration which can be obtained by using one of the selected two sets of BCs. 


\subsection{Dirichlet BCs (Type I)}

The Laplace Transformation of Eqs. (22a) and (22b) are given as

$$
\bar{c}_{i}(s, 0)=\frac{c_{\mathrm{inj}, i}\left(1-e^{-s \tau_{\mathrm{inj}}}\right)}{s}, \quad \frac{d \bar{c}_{i}}{d x}(s, \infty)=0 .
$$

On using the transformations in Eq. (47), Eq. (51) yields

$$
\begin{aligned}
& b_{1}(s, x=0)=\frac{\left(1-e^{-s \tau_{\mathrm{inj}}}\right)}{s} c_{\mathrm{inj}, 1}, \quad \frac{d b_{1}}{d x}(s, \infty)=0 . \\
& b_{2}(s, x=0)=\frac{\left(1-e^{-s \tau_{\mathrm{inj}}}\right)}{s} c_{\mathrm{inj}, 2}+\frac{\xi_{p, 2} P e_{l} A(s)}{P e_{l} \phi_{1}(s)-P e_{l} \phi_{2}(s)} b_{1}(s, x=0), \quad \frac{d b_{2}}{d x}(s, \infty)=0 .
\end{aligned}
$$

After applying these boundary conditions on Eq. (49), the values of $A_{1}$ and $B_{1}$ are obtained as

$$
A_{1}=\frac{\left(1-e^{-s \tau_{\mathrm{inj}}}\right)}{s} c_{\mathrm{inj}, 1}, \quad B_{1}=0
$$

Thus, Eqs. (47), Eq. (49) and Eq. (54) give

$$
\bar{c}_{1}(s, x)=\frac{c_{\mathrm{inj}, 1}\left(1-e^{-s \tau_{\mathrm{inj}}}\right)}{s} e^{m_{1} x} .
$$

The value of $m_{1}$ is given by Eq. (49) for the upper negative sign. Similarly, on using Eq. (53) in Eq. (50), we get the values of $A_{2}$ and $B_{2}$ as follows:

$$
A_{2}=\frac{\left(1-e^{-s \tau_{\mathrm{inj}}}\right)}{s}\left[c_{\mathrm{inj}, 2}+\frac{\xi_{p, 2} P e_{l} A(s)}{P e_{l} \phi_{1}(s)-P e_{l} \phi_{2}(s)} c_{\mathrm{inj}, 1}\right], \quad B_{2}=0 .
$$

With these values of $A_{2}$ and $B_{2}$ and using Eq. (47) in Eq. (50), we obtain

$$
\overline{c_{2}}(s, x)=\frac{c_{\mathrm{inj}, 1}\left(1-e^{-s \tau_{\mathrm{inj}}}\right)}{s}\left(\frac{\xi_{p, 2} A(s)}{\phi_{1}(s)-\phi_{2}(s)}\right)\left(e^{m_{3} x}-e^{m_{1} x}\right)+\frac{c_{\mathrm{inj}, 2}\left(1-e^{-s \tau_{\mathrm{inj}}}\right)}{s} e^{m_{3} x} .
$$

Analytical Laplace inversions are not possible to bring back solutions in the time domain

$\tau$. Therefore, the numerical inverse Laplace transformation is employed to find the original solutions $c_{j}(\tau, x)$ for $j=1,2$ [44]. In this work, an efficient numerical Laplace inversion method, based on a Fourier series expansion developed by Durbin [45], is applied as explained below. 
The solution in the time domain $c_{j}(\tau, x)$ can be obtained by using the exact formula for the back transformation as

$$
c_{j}(\tau, x)=L^{-1}\left[\bar{c}_{j}(s, x)\right]=\frac{1}{2 \pi i} \int_{v-i \infty}^{v+i \infty} e^{-t s} \bar{c}_{j}(s, x) d s, \quad j=1,2,
$$

with $s=v+i w ; v, w \in \mathbb{R}$. The real constant $v$ exceeds the real part of all the singularities of $\bar{c}_{j}(s, x)$. The integrals in Eqs. (25) and (58) exist for $\operatorname{Re}(s)>\tilde{\alpha} \in \mathbb{R}$ if

(a) $c_{j}$ is locally integrable,

(b) there exist a $\tau_{0} \geq 0$ and $p, \tilde{\alpha} \in \mathbb{R}$, such that $c_{j}(\tau, x) \leq p e^{\tilde{\alpha} \tau}$ for all $\tau \geq \tau_{0}$,

(c) for all $\tau \in(0, \infty)$ there is a neighborhood in which $c_{j}$ is of bounded variation.

In the following we always assume that $c_{j}$ fulfils the above conditions and in addition that there are no singularities of $\bar{c}_{j}(s, x)$ to the right of the origin. Therefore, Eqs. (25) and (58) are defined for all $y>0$. The possibility to choose $v>0$ arbitrarily, is the basis of the methods of Durbin [45]. The integral in Eq. (58) is equivalently expressed in the interval $[0,2 T]$ as $[45]$

$$
c_{j}(\tau, x)=\frac{e^{v \tau}}{\pi} \int_{0}^{\infty}\left[\operatorname{Re}\left\{\bar{c}_{j}(s, x)\right\} \cos (w \tau)-\operatorname{Im}\left\{\bar{c}_{j}(s, x)\right\} \sin (w \tau)\right] d w .
$$

Durbin derived the following approximate expression for Eq. (59):

$$
\begin{aligned}
c_{j}(\tau, x)= & \frac{e^{v \tau}}{T}\left[-\frac{1}{2} \operatorname{Re}\left\{\bar{c}_{j}(v, x)\right\}+\sum_{p=0}^{\infty} \operatorname{Re}\left\{\bar{c}_{j}\left(v+i \frac{p \pi}{T}, x\right)\right\} \cos \left(\frac{p \pi \tau}{T}\right)\right. \\
& \left.-\sum_{p=0}^{\infty} \operatorname{Im}\left\{\bar{c}_{j}\left(v+i \frac{p \pi}{T}, x\right)\right\} \sin \left(\frac{p \pi \tau}{T}\right)\right] .
\end{aligned}
$$

In the numerical computations, the infinite series in Eq. (60) can only be summed up to a finite number $N_{p}$ of terms only. Thus, a truncation error occurs in the numerical computations. In this work, the numerical Laplace inversion formula in Eq. (60) is applied to obtain the time domain solution $c_{j}(\tau, x)$ by considering $N_{p}=10^{3}$. 


\subsection{Danckwerts BCs (Type II)}

The Laplace transformations of Danckwerts BCs given by Eqs. (23a) and (23b) are given as

$$
\bar{c}_{i}(s, 0)=\frac{c_{\mathrm{inj}, i}}{s}\left(1-e^{-s \tau_{\mathrm{inj}}}\right)+\left.\frac{1}{P e_{l}} \frac{d \bar{c}_{i}}{d x}\right|_{x=0}, \quad \frac{d \bar{c}_{i}}{d x}(s, x=1)=0, \quad i=1,2 .
$$

Following the same solution procedure of Subsection 3.1, the Laplace domain solutions can be obtained as

$$
\begin{aligned}
\bar{c}_{1}(s, x)= & \frac{c_{\mathrm{inj}, 1}\left(1-e^{-s \tau_{\mathrm{inj}}}\right)}{s} \frac{\left(m_{2}-m_{1}\right) e^{m_{2}+m_{1}}}{\left(1-\frac{m_{1}}{P e_{l}}\right) m_{2} e^{m_{2}}-\left(1-\frac{m_{2}}{P e_{l}}\right) m_{1} e^{m_{1}}}, \\
\bar{c}_{2}(s, x)= & \frac{-\xi_{p, 2} A(s)}{\phi_{1}(s)-\phi_{2}(s)} \frac{c_{\mathrm{inj}, 1}\left(1-e^{-s \tau_{\mathrm{inj}}}\right)}{s} \frac{\left(m_{2}-m_{1}\right) e^{m_{2}+m_{1}}}{\left(1-\frac{m_{1}}{P e_{l}}\right) m_{2} e^{m_{2}}-\left(1-\frac{m_{2}}{P e_{l}}\right) m_{1} e^{m_{1}}} \\
& +\frac{\left(1-e^{-s \tau_{\mathrm{inj}}}\right)}{s} \frac{\left[c_{\mathrm{inj}, 2}-\left(\frac{-\xi_{p, 2} F A(s)}{\phi_{1}(s)-\phi_{2}(s)}\right) c_{\mathrm{inj}, 1}\right]\left(m_{3}-m_{4}\right) e^{\left(m_{4}+m_{3}\right)}}{\left(1-\frac{m_{4}}{P e_{l}}\right) m_{3} e^{m_{3}}-\left(1-\frac{m_{3}}{P e_{l}}\right) m_{4} e^{m_{4}}} .
\end{aligned}
$$

There is no possibility to apply analytical back transformation on these Laplace domain solutions. However, the numerical Laplace inversion can be applied to obtain a discrete solution in time. In this technique, the integral of inverse Laplace transformation is approximated by Fourier series [44, 45].

This completes the derivation of analytical solutions for Irreversible two-component linear RGRM.

\section{RGRM for reversible Reaction $A \rightleftharpoons B$}

Now, we present the general case of linear reversible reaction carried out in a chromatographic reactor. In this case, the component $A$ (component 1 ) is injected to the column which converts to component $B$ (component 2) with a reaction rate constant $\nu_{1}$. Because

of the reversibility of the reaction, component $B$ also converts partly back to component $A$ with a reaction rate constant $\nu_{2}$. The model equations for transport in the bulk of liquid are formulated as

$$
\begin{aligned}
\frac{\partial c_{1}}{\partial t}+u \frac{\partial c_{1}}{\partial z} & =D_{z} \frac{\partial^{2} c_{1}}{\partial z^{2}}-\frac{3}{R_{p}} F k_{\mathrm{ext}, 1}\left(c_{1}-\left.c_{p, 1}\right|_{r=R_{p}}\right) \\
\frac{\partial c_{2}}{\partial t}+u \frac{\partial c_{2}}{\partial z} & =D_{z} \frac{\partial^{2} c_{2}}{\partial z^{2}}-\frac{3}{R_{p}} F k_{\mathrm{ext}, 2}\left(c_{2}-\left.c_{p, 2}\right|_{r=R_{p}}\right) .
\end{aligned}
$$


For dynamics in the particle macropores, the model equations are expressed as

$$
\begin{aligned}
\epsilon_{p} \frac{\partial c_{p, 1}}{\partial t}+\left(1-\epsilon_{p}\right) \frac{\partial q_{p, 1}^{*}}{\partial t}= & \frac{1}{r^{2}} \frac{\partial}{\partial r}\left[r^{2}\left(\epsilon_{p} D_{p, 1} \frac{\partial c_{p, 1}}{\partial r}+\left(1-\epsilon_{p}\right) D_{s, 1} \frac{\partial q_{p, 1}^{*}}{\partial r}\right)\right] \\
& -\left(1-\epsilon_{p}\right) \nu_{1} q_{p, 1}^{*}+\left(1-\epsilon_{p}\right) \nu_{2} q_{p, 2}^{*}, \\
\epsilon_{p} \frac{\partial c_{p, 2}}{\partial t}+\left(1-\epsilon_{p}\right) \frac{\partial q_{p, 2}^{*}}{\partial t}= & \frac{1}{r^{2}} \frac{\partial}{\partial r}\left[r^{2}\left(\epsilon_{p} D_{p, 2} \frac{\partial c_{p, 2}}{\partial r}+\left(1-\epsilon_{p}\right) D_{s, 2} \frac{\partial q_{p, 2}^{*}}{\partial r}\right)\right] \\
& +\left(1-\epsilon_{p}\right) \nu_{1} q_{p, 1}^{*}-\left(1-\epsilon_{p}\right) \nu_{2} q_{p, 2}^{*},
\end{aligned}
$$

where $q_{p, i}^{*}(i=1,2)$ are given by Eq. (7). On using the dimensionless variables in Eq. (13) and isotherms $q_{p, i}^{*}$ in Eq. (7), the above equations can be rewritten as

$$
\begin{aligned}
\frac{\partial c_{1}}{\partial \tau}+\frac{\partial c_{1}}{\partial x} & =\frac{1}{P e_{l}} \frac{\partial^{2} c_{1}}{\partial x^{2}}-\xi_{p, 1}\left(c_{1}-\left.c_{p, 1}\right|_{\rho=1}\right), \\
\frac{\partial c_{2}}{\partial \tau}+\frac{\partial c_{2}}{\partial x} & =\frac{1}{P e_{l}} \frac{\partial^{2} c_{1}}{\partial x^{2}}-\xi_{p, 2}\left(c_{2}-\left.c_{p, 2}\right|_{\rho=1}\right), \\
a_{1}^{*} \frac{\partial}{\partial \tau}\left(\rho c_{p, 1}\right) & =\eta_{p, 1} \frac{\partial^{2}}{\partial \rho^{2}}\left(\rho c_{p, 1}\right)-\left(1-\epsilon_{p}\right) \omega_{1}\left(\rho c_{p, 1}\right)+\left(1-\epsilon_{p}\right) \omega_{2}\left(\rho c_{p, 2}\right), \\
a_{2}^{*} \frac{\partial}{\partial \tau}\left(\rho c_{p, 2}\right) & =\eta_{p, 2} \frac{\partial^{2}}{\partial \rho^{2}}\left(\rho c_{p, 2}\right)+\left(1-\epsilon_{p}\right) \omega_{1}\left(\rho c_{p, 2}\right)-\left(1-\epsilon_{p}\right) \omega_{2}\left(\rho c_{p, 1}\right) .
\end{aligned}
$$

The same initial and boundary conditions are used as given by Eqs. (20)-(24). The application of Laplace transformation on the above equations yields

$$
\begin{aligned}
& s \overline{c_{1}}+\frac{d \overline{c_{1}}}{d x}=\frac{1}{P e_{l}} \frac{d^{2} \overline{c_{1}}}{d x^{2}}-\xi_{p, 1}\left(\overline{c_{1}}-\left.c_{p, 1}^{-}\right|_{\rho=1}\right), \\
& s \overline{c_{2}}+\frac{d \overline{c_{2}}}{d x}=\frac{1}{P e_{l}} \frac{d^{2} \overline{c_{2}}}{d x^{2}}-\xi_{p, 2}\left(\overline{c_{2}}-\left.\bar{c}_{p, 2}^{-}\right|_{\rho=1}\right), \\
& \frac{d^{2}}{d \rho^{2}}\left(\rho \bar{c}_{p, 1}\right)-\frac{a_{1}^{*} s}{\eta_{p, 1}}\left(\rho \bar{c}_{p, 1}\right)-\frac{\left(1-\epsilon_{p}\right) \omega_{1}}{\eta_{p, 1}}\left(\rho \bar{c}_{p, 1}\right)+\frac{\left(1-\epsilon_{p}\right) \omega_{2}}{\eta_{p, 1}}\left(\rho \bar{c}_{p, 2}\right)=0, \\
& \frac{d^{2}}{d \rho^{2}}\left(\rho \bar{c}_{p, 2}\right)-\frac{a_{2}^{*} s}{\eta_{p, 2}}\left(\rho \bar{c}_{p, 2}\right)+\frac{\left(1-\epsilon_{p}\right) \omega_{1}}{\eta_{p, 2}}\left(\rho \bar{c}_{p, 1}\right)-\frac{\left(1-\epsilon_{p}\right) \omega_{2}}{\eta_{p, 2}}\left(\rho \bar{c}_{p, 2}\right)=0 .
\end{aligned}
$$

In matrix natation, Eqs. (75) and Eq. (74) can be expressed as

$$
\frac{d^{2}}{d x^{2}}\left(\begin{array}{c}
\rho \bar{c}_{p, 1} \\
\rho \bar{c}_{p, 2}
\end{array}\right)-\left[\begin{array}{cc}
\frac{a_{1}^{*} s+\left(1-\epsilon_{p}\right) \omega_{1}}{\eta_{p, 1}} & -\frac{\left(1-\epsilon_{p}\right) \omega_{2}}{\eta_{p, 1}} \\
-\frac{\left(1-\epsilon_{p}\right) \omega_{1}}{\eta_{p, 2}} & \frac{a_{2}^{*} s+\left(1-\epsilon_{p}\right) \omega_{2}}{\eta_{p, 2}}
\end{array}\right]\left(\begin{array}{c}
\rho \bar{c}_{1} \\
\rho \bar{c}_{2}
\end{array}\right)=\left(\begin{array}{l}
0 \\
0
\end{array}\right) .
$$


The reaction coefficient matrix $\left[B^{\prime}\right]$ of Eq. (76) is given as

$$
B^{\prime}=\left[\begin{array}{cc}
\frac{a_{1}^{*} s+\left(1-\epsilon_{p}\right) \omega_{1}}{\eta_{p, 1}} & -\frac{\left(1-\epsilon_{p}\right) \omega_{2}}{\eta_{p, 1}} \\
-\frac{\left(1-\epsilon_{p}\right) \omega_{1}}{\eta_{p, 2}} & \frac{a_{2}^{*} s+\left(1-\epsilon_{p}\right) \omega_{2}}{\eta_{p, 2}}
\end{array}\right]
$$

The eigenvalues and eigenvectors of the matrix $\left[B^{\prime}\right]$ are given as:

$$
\lambda_{1,2}^{\prime}=\frac{\beta_{1} \pm \sqrt{\beta_{1}^{2}-4 \beta_{2}}}{2} \quad x_{1,2}^{\prime}=\left(\begin{array}{c}
\lambda_{1,2}^{\prime}-\frac{a_{2}^{*} s+\left(1-\epsilon_{p}\right) \omega_{2}}{\eta_{p, 2}} \\
-\frac{\left(1-\epsilon_{p}\right) \omega_{1}}{\eta_{p, 2}}
\end{array}\right)
$$

where

$$
\begin{aligned}
& \beta_{1}(s)=\frac{a_{1}^{*} s}{\eta_{p, 1}}+\frac{a_{2}^{*} s}{\eta_{p, 2}}+\frac{\left(1-\epsilon_{p}\right) \omega_{1}}{\eta_{p, 1}}+\frac{\left(1-\epsilon_{p}\right) \omega_{2}}{\eta_{p, 2}}, \\
& \beta_{2}(s)=\frac{a_{1}^{*} a_{2}^{*} s^{2}}{\eta_{p, 1} \eta_{p, 2}}+\frac{a_{2}^{*} s\left(1-\epsilon_{p}\right) \omega_{1}}{\eta_{p, 1} \eta_{p, 2}}+\frac{a_{1}^{*} s\left(1-\epsilon_{p}\right) \omega_{2}}{\eta_{p, 1} \eta_{p, 2}} .
\end{aligned}
$$

Thus, we have the following linear transformation

$$
\left(\begin{array}{c}
\bar{c}_{p, 1} \\
\bar{c}_{p, 2}
\end{array}\right)=\left[\begin{array}{cc}
\lambda_{1}^{\prime}-\frac{a_{2}^{*} s+\left(1-\epsilon_{p}\right) \omega_{2}}{\eta_{p, 2}} & \lambda_{2}^{\prime}-\frac{a_{2}^{*} s+\left(1-\epsilon_{p}\right) \omega_{2}}{\eta_{p, 2}} \\
-\frac{\left(1-\epsilon_{p}\right) \omega_{1}}{\eta_{p, 2}} & -\frac{\left(1-\epsilon_{p}\right) \omega_{1}}{\eta_{p, 2}}
\end{array}\right]\left(\begin{array}{c}
\bar{b}_{p, 1} \\
\bar{b}_{p, 2}
\end{array}\right)
$$

Applying the above linear transformation on Eq. (76), we get

$$
\frac{d^{2}}{d x^{2}}\left(\begin{array}{c}
\rho \bar{b}_{p, 1} \\
\rho \bar{b}_{p, 2}
\end{array}\right)-\left[\begin{array}{cc}
\lambda_{1}^{\prime} & 0 \\
0 & \lambda_{2}^{\prime}
\end{array}\right]\left(\begin{array}{c}
\rho \bar{b}_{p, 1} \\
\rho \bar{b}_{p, 2}
\end{array}\right)=\left(\begin{array}{l}
0 \\
0
\end{array}\right)
$$

Eq. (82) represents a system of two independent ODEs. Their explicit solutions are given as

$$
\bar{b}_{p, 1}(s, x, \rho)=\frac{1}{\rho}\left[A_{1}^{\prime} e^{\sqrt{\lambda_{1}^{\prime}} \rho}+B_{1}^{\prime} e^{-\sqrt{\lambda_{1}^{\prime}} \rho}\right], \quad \bar{b}_{p, 2}(s, x, \rho)=\frac{1}{\rho}\left[A_{2}^{\prime} e^{\sqrt{\lambda_{2}^{\prime}} \rho}+B_{2}^{\prime} e^{-\sqrt{\lambda_{2}^{\prime}} \rho}\right] .
$$

From Eqs. (24) and (81), one can easily find

$$
\left.\frac{\partial b_{p, i}(s, \rho)}{\partial \rho}\right|_{\rho=0}=0, \quad i=1,2
$$

On using the above boundary conditions, we get from Eq. (83) $B_{1}^{\prime}=-A_{1}^{\prime}$ and $B_{2}^{\prime}=A_{2}^{\prime}$. Thus, Eq. (83) reduces to

$$
b_{p, 1}(s, x, \rho)=\frac{2 A_{1}^{\prime}}{\rho} \sinh \left(\sqrt{\lambda_{1}^{\prime}} \rho\right), \quad b_{p, 2}(s, x, \rho)=\frac{2 A_{2}^{\prime}}{\rho} \sinh \left(\sqrt{\lambda_{2}^{\prime}} \rho\right) .
$$


By using the transformation in Eq. (81) and values of $b_{i}$ in Eq. (85), we obtain

$$
\begin{aligned}
c_{p, 1}(s, x, \rho) & =\left[\lambda_{1}^{\prime}-\frac{a_{2}^{*} s+\left(1-\epsilon_{p}\right) \omega_{2}}{\eta_{p, 2}}\right] \frac{2 A_{1}^{\prime}}{\rho} \sinh \left(\sqrt{\lambda_{1}^{\prime}} \rho\right) \\
& +\left[\lambda_{2}^{\prime}-\frac{a_{2}^{*} s+\left(1-\epsilon_{p}\right) \omega_{2}}{\eta_{p, 2}}\right] \frac{2 A_{2}^{\prime}}{\rho} \sinh \left(\sqrt{\lambda_{2}^{\prime}} \rho\right), \\
c_{p, 2}(s, x, \rho) & =-\frac{\left(1-\epsilon_{p}\right) \omega_{1}}{\eta_{p, 2}}\left[\frac{2 A_{1}^{\prime}}{\rho} \sinh \left(\sqrt{\lambda_{1}^{\prime}} \rho\right)+\frac{2 A_{2}^{\prime}}{\rho} \sinh \left(\sqrt{\lambda_{2}^{\prime}} \rho\right)\right] .
\end{aligned}
$$

By using second boundary condition at $\rho=1$ (c.f. Eq. (24)) in Eqs. (86) and (87), we get a system of two equations in term of $A_{1}^{\prime}$ and $A_{2}^{\prime}$. On solving those equations, we obtain

$$
\begin{aligned}
& A_{1}^{\prime}=\frac{1}{2 \sinh \left(\sqrt{\lambda_{1}^{\prime}}\right)}\left[\frac{\alpha_{1}(s)}{A(s)} \bar{c}_{1}+\frac{\alpha_{2}(s)}{A(s)} \frac{\left.\eta_{p, 2}\left(\lambda_{2}^{\prime}-\frac{a_{2}^{*} s+\left(1-\epsilon_{p}\right) \omega_{2}}{\eta_{p, 2}}\right)\right)}{\left(1-\epsilon_{p}\right) \omega_{1}} \bar{c}_{2}\right], \\
& A_{2}^{\prime}=-\frac{1}{2 \sinh \left(\sqrt{\lambda_{2}^{\prime}}\right)}\left[\frac{\alpha_{3}(s)}{A(s)} \bar{c}_{1}+\frac{\alpha_{4}(s)}{A(s)} \frac{\left.\eta_{p, 2}\left(\lambda_{1}^{\prime}-\frac{a_{2}^{*} s+\left(1-\epsilon_{p}\right) \omega_{2}}{\eta_{p, 2}}\right)\right)}{\left(1-\epsilon_{p}\right) \omega_{1}} \bar{c}_{2}\right],
\end{aligned}
$$

where

$$
\begin{aligned}
\alpha_{1}(s)= & B i_{p, 1}\left[B i_{p, 2}-1+\sqrt{\lambda_{2}^{\prime}} \operatorname{coth}\left(\sqrt{\lambda_{2}^{\prime}}\right)\right], \quad \alpha_{2}(s)=B i_{p, 2}\left[B i_{p, 1}-1+\sqrt{\lambda_{2}^{\prime}} \operatorname{coth}\left(\sqrt{\lambda_{2}^{\prime}}\right)\right], \\
\alpha_{3}(s)= & B i_{p, 1}\left[B i_{p, 2}-1+\sqrt{\lambda_{1}^{\prime}} \operatorname{coth}\left(\sqrt{\lambda_{1}^{\prime}}\right)\right], \quad \alpha_{4}(s)=B i_{p, 2}\left[B i_{p, 1}-1+\sqrt{\lambda_{1}^{\prime}} \operatorname{coth}\left(\sqrt{\lambda_{1}^{\prime}}\right)\right], \\
A(s)= & \left(\lambda_{1}^{\prime}-\frac{a_{2}^{*} s+\left(1-\epsilon_{p}\right) \omega_{2}}{\eta_{p, 2}}\right)\left[B i_{p, 1}-1+\sqrt{\lambda_{1}^{\prime}} \operatorname{coth}\left(\sqrt{\lambda_{1}^{\prime}}\right)\right]\left[B i_{p, 2}-1+\sqrt{\lambda_{2}^{\prime}} \operatorname{coth}\left(\sqrt{\lambda_{2}^{\prime}}\right)\right] \\
& -\left(\lambda_{2}^{\prime}-\frac{a_{2}^{*} s+\left(1-\epsilon_{p}\right) \omega_{2}}{\eta_{p, 2}}\right)\left[B i_{p, 1}-1+\sqrt{\lambda_{2}^{\prime}} \operatorname{coth}\left(\sqrt{\lambda_{2}^{\prime}}\right)\right]\left[B i_{p, 2}-1+\sqrt{\lambda_{1}^{\prime}} \operatorname{coth}\left(\sqrt{\lambda_{1}^{\prime}}\right)\right] .
\end{aligned}
$$

Thus, Eqs. (86) and (87) take the forms

$$
\begin{aligned}
\left.c_{p, 1}\right|_{\rho=1}= & {\left.\left.\left[\frac{\alpha_{1}(s)}{A(s)}\left(\lambda_{1}^{\prime}-\frac{a_{2}^{*} s+\left(1-\epsilon_{p}\right) \omega_{2}}{\eta_{p, 2}}\right)\right)-\frac{\alpha_{3}(s)}{A(s)}\left(\lambda_{2}^{\prime}-\frac{a_{2}^{*} s+\left(1-\epsilon_{p}\right) \omega_{2}}{\eta_{p, 2}}\right)\right)\right] \bar{c}_{1} } \\
& -\frac{\left(1-\epsilon_{p}\right) \omega_{2}}{\eta_{p, 1}}\left[\frac{\alpha_{2}(s)}{A(s)}-\frac{\alpha_{4}(s)}{A(s)}\right] \bar{c}_{2}, \\
\left.c_{p, 2}\right|_{\rho=1}= & -\frac{\left(1-\epsilon_{p}\right) \omega_{1}}{\eta_{p, 2}}\left[\frac{\alpha_{1}(s)}{A(s)}-\frac{\alpha_{3}(s)}{A(s)}\right] \bar{c}_{1} \\
& \left.\left.+\left[\frac{\alpha_{4}(s)}{A(s)}\left(\lambda_{1}^{\prime}-\frac{a_{2}^{*} s+\left(1-\epsilon_{p}\right) \omega_{2}}{\eta_{p, 2}}\right)\right)-\frac{\alpha_{2}(s)}{A(s)}\left(\lambda_{2}^{\prime}-\frac{a_{2}^{*} s+\left(1-\epsilon_{p}\right) \omega_{2}}{\eta_{p, 2}}\right)\right)\right] \bar{c}_{2} .
\end{aligned}
$$

By using Eqs. (93) and (94) in Eqs. (72) and (73), we get the following system of ODEs

$$
\frac{d^{2}}{d x^{2}}\left(\begin{array}{l}
\bar{c}_{1} \\
\bar{c}_{2}
\end{array}\right)-P e_{l} \frac{d}{d x}\left(\begin{array}{c}
\bar{c}_{1} \\
\bar{c}_{2}
\end{array}\right)+\left[\begin{array}{cc}
-r_{1}-s P e_{l} & r_{2} \\
r_{3} & -r_{4}-s P e_{l}
\end{array}\right]\left(\begin{array}{l}
\bar{c}_{1} \\
\bar{c}_{2}
\end{array}\right)=\left(\begin{array}{l}
0 \\
0
\end{array}\right)
$$


where

$$
\begin{aligned}
& \left.\left.r_{1}=P e_{l} \xi_{p, 1}\left[1-\frac{\alpha_{1}(s)}{A(s)}\left(\lambda_{1}^{\prime}-\frac{a_{2}^{*} s+\left(1-\epsilon_{p}\right) \omega_{2}}{\eta_{p, 2}}\right)\right)+\frac{\alpha_{3}(s)}{A(s)}\left(\lambda_{2}^{\prime}-\frac{a_{2}^{*} s+\left(1-\epsilon_{p}\right) \omega_{2}}{\eta_{p, 2}}\right)\right)\right], \\
& r_{2}=-P e_{l} \xi_{p, 1} \frac{\left(1-\epsilon_{p}\right) \omega_{2}}{\eta_{p, 1}}\left[\frac{\alpha_{2}(s)}{A(s)}-\frac{\alpha_{4}(s)}{A(s)}\right], \\
& r_{3}=-P e_{l} \xi_{p, 2} \frac{\left(1-\epsilon_{p}\right) \omega_{1}}{\eta_{p, 2}}\left[\frac{\alpha_{1}(s)}{A(s)}-\frac{\alpha_{3}(s)}{A(s)}\right], \\
& \left.\left.r_{4}=P e_{l} \xi_{p, 2}\left[1-\frac{\alpha_{4}(s)}{A(s)}\left(\lambda_{1}^{\prime}-\frac{a_{2}^{*} s+\left(1-\epsilon_{p}\right) \omega_{2}}{\eta_{p, 2}}\right)\right)+\frac{\alpha_{2}(s)}{A(s)}\left(\lambda_{2}^{\prime}-\frac{a_{2}^{*} s+\left(1-\epsilon_{p}\right) \omega_{2}}{\eta_{p, 2}}\right)\right)\right] .
\end{aligned}
$$

By adopting the same decoupling procedure, we get the following Laplace domain solutions of the system in Eq. (95) for the considered two sets of BCs.

\subsection{Type 1: Dirichlet BCs}

In this case, the boundary conditions in Eqs. (22a), (22b) are taken into account. The Laplace domain solutions are given as

$$
\begin{aligned}
\bar{c}_{1}(s, x)= & \frac{\left(1-e^{-s \tau_{\mathrm{inj}}}\right) \lambda_{3}^{\prime \prime}\left(r_{3} c_{\mathrm{inj}, 1}-\lambda_{4}^{\prime \prime} c_{\mathrm{inj}, 2}\right)\left(m_{2}^{\prime} e^{m_{2}^{\prime}+m_{1}^{\prime} x}-m_{1}^{\prime} e^{m_{1}^{\prime}+m_{2}^{\prime} x}\right)}{s r_{3}\left(\lambda_{3}^{\prime \prime}-\lambda_{4}^{\prime \prime}\right)\left(m_{2}^{\prime} e^{m_{2}^{\prime}}-m_{1}^{\prime} e^{m_{1}^{\prime}}\right)} \\
& -\frac{\left(1-e^{-s \tau_{\mathrm{inj}}}\right) \lambda_{4}^{\prime \prime}\left(r_{3} c_{\mathrm{inj}, 1}-\lambda_{3}^{\prime \prime} c_{\mathrm{inj}, 2}\right)\left(m_{4}^{\prime} e^{m_{4}^{\prime}+m_{3}^{\prime} x}-m_{3}^{\prime} e^{m_{3}^{\prime}+m_{4}^{\prime} x}\right)}{s r_{3}\left(\lambda_{3}^{\prime \prime}-\lambda_{4}^{\prime \prime}\right)\left(m_{4}^{\prime} e^{m_{4}^{\prime}}-m_{3}^{\prime} e^{m_{3}^{\prime}}\right)}, \\
\bar{c}_{2}(s, x)= & \frac{\left(1-e^{-s \tau_{\mathrm{inj}}}\right)\left(r_{3} c_{\mathrm{inj}, 1}-\lambda_{4}^{\prime \prime} c_{\mathrm{inj}, 2}\right)\left(m_{2}^{\prime} e^{m_{2}^{\prime}+m_{1}^{\prime} x}-m_{1}^{\prime} e^{m_{1}^{\prime}+m_{2}^{\prime} x}\right)}{s\left(\lambda_{3}^{\prime \prime}-\lambda_{4}^{\prime \prime}\right)\left(m_{2}^{\prime} e^{m_{2}^{\prime}}-m_{1}^{\prime} e^{m_{1}^{\prime}}\right)} \\
& -\frac{\left(1-e^{-s \tau_{\mathrm{inj}}}\right)\left(r_{3} c_{\mathrm{inj}, 1}-\lambda_{3}^{\prime \prime} c_{\mathrm{inj}, 2}\right)\left(m_{4}^{\prime} e^{m_{4}^{\prime}+m_{3}^{\prime} x}-m_{3}^{\prime} e^{m_{3}^{\prime}+m_{4}^{\prime} x}\right)}{s\left(\lambda_{3}^{\prime \prime}-\lambda_{4}^{\prime \prime}\right)\left(m_{4}^{\prime} e^{m_{4}^{\prime}}-m_{3}^{\prime} e^{m_{3}^{\prime}}\right)},
\end{aligned}
$$

where

$$
\begin{aligned}
\lambda_{1,2}^{\prime \prime} & =-\frac{1}{2}\left[r_{1}+r_{4}+2 s P e_{l} \mp \sqrt{4\left(r_{2} r_{3}-r_{1} r_{4}\right)+\left(r_{1}+r_{4}\right)^{2}}\right] \\
\lambda_{3,4}^{\prime \prime} & =-\frac{1}{2}\left[r_{1}-r_{4} \mp \sqrt{4\left(r_{2} r_{3}-r_{1} r_{4}\right)+\left(r_{1}+r_{4}\right)^{2}}\right] . \\
m_{1,2}^{\prime} & =\frac{P e_{l} \pm \sqrt{P e_{l}^{2}-4 \lambda_{1}^{\prime \prime}}}{2}, \quad m_{3,4}^{\prime}=\frac{P e_{l} \pm \sqrt{P e_{l}^{2}-4 \lambda_{2}^{\prime \prime}}}{2} .
\end{aligned}
$$

Analytical Laplace inversions of the above equations are very difficult. Therefore, numerical Laplace inversions are used to get back solutions in actual time domain [20, 34, 44, 45].

\section{Type 2: Danckwerts BCs}

In this case, the BCs in Eq. (23a) and (23b) are taken into account. The general solutions in the Laplace domain are given as 


$$
\begin{aligned}
\bar{c}_{1}(s, x) & =\frac{\left(1-e^{-s \tau_{\mathrm{inj}}}\right) \lambda_{3}^{\prime \prime}\left(r_{3} c_{\mathrm{inj}, 1}-\lambda_{4}^{\prime \prime} c_{\mathrm{inj}, 2}\right)\left(m_{1}^{\prime} e^{m_{1}^{\prime}+m_{2}^{\prime} x}-m_{2}^{\prime} e^{m_{2}^{\prime}+m_{1}^{\prime} x}\right)}{r_{3} s\left(\lambda_{3}^{\prime \prime}-\lambda_{4}^{\prime \prime}\right)\left[\left(1-\frac{m_{2}^{\prime}}{P e_{l}}\right) m_{1}^{\prime} e^{m_{1}^{\prime}}-\left(1-\frac{m_{1}^{\prime}}{P e_{l}}\right) m_{2}^{\prime} e^{m_{2}^{\prime}}\right]} \\
- & \frac{\left(1-e^{-s \tau_{\mathrm{inj}}}\right) \lambda_{4}^{\prime \prime}\left(r_{3} c_{\mathrm{inj}, 1}-\lambda_{3}^{\prime \prime} c_{\mathrm{inj}, 2}\right)\left(m_{3}^{\prime} e^{m_{3}^{\prime}+m_{4}^{\prime} x}-m_{4}^{\prime} e^{m_{4}^{\prime}+m_{3}^{\prime} x}\right)}{r_{3} s\left(\lambda_{3}^{\prime \prime}-\lambda_{4}^{\prime \prime}\right)\left[\left(1-\frac{m_{4}^{\prime}}{P e_{l}}\right) m_{3}^{\prime} e^{m_{3}^{\prime}}-\left(1-\frac{m_{3}^{\prime}}{P e_{l}}\right) m_{4}^{\prime} e^{m_{4}^{\prime}}\right]} \\
\bar{c}_{2}(s, x) & =\frac{\left(1-e^{-s \tau_{\mathrm{inj}}}\right)\left(r_{3} c_{\mathrm{inj}, 1}-\lambda_{4}^{\prime \prime} c_{\mathrm{inj}, 2}\right)\left(m_{1}^{\prime} e^{m_{1}^{\prime}+m_{2}^{\prime} x}-m_{2}^{\prime} e^{m_{2}^{\prime}+m_{1}^{\prime} x}\right)}{s\left(\lambda_{3}^{\prime \prime}-\lambda_{4}^{\prime \prime}\right)\left[\left(1-\frac{m_{2}^{\prime}}{P e_{l}}\right) m_{1}^{\prime} e^{m_{1}^{\prime}}-\left(1-\frac{m_{1}^{\prime}}{P e_{l}}\right) m_{2}^{\prime} e^{m_{2}^{\prime}}\right]} \\
- & \frac{\left(1-e^{-s \tau_{\mathrm{inj}}}\right)\left(r_{3} c_{\mathrm{inj}, 1}-\lambda_{3}^{\prime \prime} c_{\mathrm{inj}, 2}\right)\left(m_{3}^{\prime} e^{m_{3}^{\prime}+m_{4}^{\prime} x}-m_{4}^{\prime} e^{m_{4}^{\prime}+m_{3}^{\prime} x}\right)}{s\left(\lambda_{3}^{\prime \prime}-\lambda_{4}^{\prime \prime}\right)\left[\left(1-\frac{m_{4}^{\prime}}{P e_{l}}\right) m_{3}^{\prime} e^{m_{3}^{\prime}}-\left(1-\frac{m_{3}^{\prime}}{P e_{l}}\right) m_{4}^{\prime} e^{m_{4}^{\prime}}\right]}
\end{aligned}
$$

Here, $\lambda_{i}^{\prime \prime}$ and $m_{i}^{\prime}$ for $i=1,2,3,4$ are given by Eqs. (102)-(104). Once again, analytical Laplace inversions of the above equations are not possible. Therefore, numerical Laplace inversions are used to get back the time domain solutions $[20,34,44,45]$.

\section{Moments analysis}

Moment analysis is an attractive technique for deducing important information about the retention equilibrium and mass transfer kinetics in the column. This approach has been found instructive in the literature $[3,19,35]$. A set of statistical temporal moments can define the appearance of the plotted elution profile. For example, the appropriate forms of the first, second and third will describe the mean, spread and skew, respectively, of the distribution.

In this article, we have derived the first two analytical temporal moments of the Laplace domain solutions (c.f Eqs. (55) and (57)) for irreversible reaction considering Dirichlet BCs and $c_{\mathrm{inj}, 2}=0$, i.e. considering the injection of component 1 (reactant) only. These moments were derived using the following moment generating property of the Laplace domain solutions.

The zeroth moments [26] are defined as

$$
\mu_{0}^{(i)}=\frac{L}{u} \lim _{s \rightarrow 0}\left(\bar{c}_{i}(s, x=1)\right), \quad i=1,2
$$

and the $n$-th moments are given as

$$
\mu_{n}^{(i)}=\left(-\frac{L}{u}\right)^{n} \frac{1}{\lim _{s \rightarrow 0}\left(\bar{c}_{i}(s, x=1)\right)} \lim _{s \rightarrow 0} \frac{d^{n}\left(\bar{c}_{i}(s, x=1)\right)}{d s^{n}}, \quad n=1,2,3, \cdots .
$$

The resulting expressions of the moments are presented in Appendix A. The derivation of analytical moments for reversible reaction were very difficult due to their lengthy expressions. For that reason, the numerical temporal moments up to order three are also presented in the case studies. 
The normalized numerical moments can be obtained by integrating the band profiles at the outlet of a chromatographic column as

$$
\mu_{0}^{(i)}=\frac{L}{u} \int_{0}^{\infty} c_{i}(\tau, x=1) d \tau, \quad \mu_{n}^{(i)}=\left(\frac{L}{u}\right)^{n} \frac{\int_{0}^{\infty} c_{i}(\tau, x=1) \tau^{n} d \tau}{\int_{0}^{\infty} c_{i}(\tau, x=1) d \tau}, \quad n=1,2, \cdots .
$$

While, the $n$-th central moments are expressed as

$$
\mu_{n}^{\prime(i)}=\left(\frac{L}{u}\right)^{n} \frac{\int_{0}^{\infty} c_{i}(\tau, x=1)\left(\tau-\frac{u}{L} \mu_{1}^{(i)}\right)^{n} d \tau}{\int_{0}^{\infty} c(\tau, x=1) d \tau}, \quad n=2,3, \cdots, \quad i=1,2 .
$$

In the discussion of test problems, a comparison first three moments is given which were obtained by integrating the concentration profiles of analytical solutions and numerical solutions of finite volume scheme [40]. The trapezoidal rule is applied to numerically approximate the integral terms appearing in the above equations.

\section{Numerical case studies}

The derived semi-analytical solutions presented in the aforementioned sections are analyzed by considering several test problems. For validation, the derived semi-analytical solutions of twocomponent RGRM are compared with the numerical solutions of the same model. These numerical solutions are obtained by implementing a high resolution finite volume scheme(HR-FVS) of Koren [39, 40]. A complete set of basic model parameters used in the test problems is given in Table 1. To derive reasonable (prototypic) results the values were chosen in typical ranges for these parameters. Here, we have assumed that $D_{\mathrm{eff}, i}$ and $k_{\mathrm{ext}, i}$ are the same for all components.

\subsection{Problem 1: Linear irreversible reaction}

In this section, the semi-analytical and numerical solutions of the model equations Eqs. (1)-(4) are compared for the considered irreversible reaction in the solid phase. The results obtained demonstrate the effects of boundary conditions, reaction rate constant $\omega_{1}$, Peclet number $P e_{l}$, film mass transfer resistance $B i_{p}$, and intraparticle diffusion resistance $\eta_{p}$ on the concentration profiles and moments.

Figure 1 illustrates the effects of injected sample volume on the concentration profiles. The concentration profiles are plotted at the column outlet after injecting a rectangular pulse of finite width in an empty column $\left(c_{i, \text { init }}=0 \mathrm{~g} / \mathrm{l}\right.$ for $\left.i=1,2\right)$ using Dirichlet BCs. In Figure 1(a), equal 
amount of injections are considered for both components (i.e. $c_{\mathrm{inj}, 1}=0.5 \mathrm{~g} / \mathrm{l}, c_{\mathrm{inj}, 2}=0.5 \mathrm{~g} / \mathrm{l}$ ) along with the reaction rate constant $\omega_{1}=0.1$. The amount of product (component 2 ) increases due to the conversion of reactant (component 1) into the product through irreversible reaction. On the other hand, in Figure 1(b), the amount of injection for reactant (component 1) is $c_{\mathrm{inj}, 1}=0.5 \mathrm{~g} / \mathrm{l}$ and for the product (component 2) is $c_{\mathrm{inj}, 1}=0.25 \mathrm{~g} / \mathrm{l}$. Once gain, the same amount of reactant (component 1) is converted into the product (component 2) but the total amount of product is proportional to the amount of converted reactant plus the amount of product injected. Due to the less amount of injected product, the hight of component 2 in Figure 1(b) is smaller than that in Figure 1(a). Moreover, it can observed that component 1 elutes later than component 2 due to a difference in their affinities (i.e $a_{1}=2.5$ and $a_{2}=0.5$ ). In both cases, the semi-analytical and numerical solutions are in good agreement with each other.

Figure 2 demonstrates the effects of boundary conditions on the concentration profiles of component 1 and component 2. The concentration profiles at the column outlet are plotted for different values of $P e_{l}$ considering $c_{\mathrm{inj}, 1}=0.5 \mathrm{~g} / \mathrm{l}, c_{\mathrm{inj}, 2}=0.5 \mathrm{~g} / \mathrm{l}, c_{i, \text { init }}=0.0 \mathrm{~g} / \mathrm{l}$ for $i=1,2$, and $\omega_{1}=0.1$. From these plots one can observe that the concentration profiles of Dirichlet and Danckwert BCs deviate from each other for $P e_{l}=1.25$. However, for large Peclet, i.e. $P e_{l}=125$, or small axial dispersion the concentration profiles obtained by Dirichlet and Danckwert BCs are almost identical. Thus, for small large values of axial dispersion coefficient, the more accurate Danckwerts BCs should be used which accounts for back mixing near the column inlet.

Figure 3 depicts the effects of reaction rate constant $\omega_{1}$ on the concentration profiles. The concentration profiles are plotted by using Danckwert BCs and taking $\omega_{1}$ as a parameter. Here, we have chosen $c_{\text {inj,1 }}=0.5 \mathrm{~g} / \mathrm{l}, c_{\mathrm{inj}, 2}=0.5 \mathrm{~g} / \mathrm{l}$, and $c_{i, \text { init }}=0.0 \mathrm{~g} / \mathrm{l}$. In this case, the amount of product increases on increasing the value of reaction rate constant, while the amount of reactant is decreasing.

Figure 4(a) displays the effects of Biot number, $B i_{p}=\frac{k_{\text {ext }} R_{p}}{D_{\mathrm{eff}_{\mathrm{i}}}}$, on the concentration profiles of both components for three different values of $B i_{p}$. For $B i_{p}=50$, the peak profiles are broadened where as for $B i_{p}=0.5$, both components have steeped profiles. Figure 4(b) examines the effects of intraparticle diffusion $\eta_{p}$ on the concentration profiles. The concentration profiles for both components are plotted for a fixed $P e_{l}=125$ and taking different values of $\eta_{p}$. For $\eta_{p}=0.02$, the column retention time reduces for both components due to slow diffusion rate, where as, 
for $\eta_{p}=20$ the retention time for both components increases. Moreover, the increasing value of $\eta_{p}$ enhances the separation of both components. Limitations in intraparticle mass transfer resistances of both components decreases the zone spreading time for the considered fully porous adsorbents.

Figure 5 gives the plots of numerical statistical moments of semi-analytical solutions for a wide range of flow rates considering Dirichlet BCs. In this case $c_{\mathrm{inj}, 1}=0.5 \mathrm{~g} / \mathrm{l}, c_{\mathrm{inj}, 2}=0.5 \mathrm{~g} / \mathrm{l}$ and $\omega_{1}=0.1$. The plots of zeroth moments show the total masses of both components. They indicate that conversion of component 1 into component 2 reduces on increasing the velocity. The plots of first moments reveal the expected linear trends of mean retention times for the reactant and product over $1 / u$. The plots of second central moments quantify the variance (spreading) of the elution profiles for both components. The third central moments describe the skewness of the elution profiles. The positive values of third central moments depict that the profiles of reactant and product are right-tailed.

Figure 5 shows the comparison of analytical (c.f. Eqs. (A-2)-(A-7)) and numerical moments for different flow rates using Dirichlet boundary conditions, $c_{\mathrm{inj}, 1}=0.5 \mathrm{~g} / \mathrm{l}, c_{\mathrm{inj}, 2}=0.0 \mathrm{~g} / \mathrm{l}$ and $\omega_{1}=0.1$. Here, the numerical moments were calculated by integrating the concentration profiles obtained through numerical Laplace inversion.

Figure 7 compares the results of reactive GRM with those of simplified reactive lumped kinetic model (LKM). The results of LKM were obtained by choosing the values of its parameters according to the relations given in Eqs. (111) and (112) [34, 46]. Here, the Reaction rate constant is taken as $\omega_{1}=0.1$. In the Figure $7(\mathrm{a})$, the first moments, i.e. the retention times, of GRM and LKM were matched through the following relation (c.f. [46])

$$
a_{i}=a_{i}^{*}, \quad i=1,2 .
$$

To match the second central moments of GRM and LKM, the following relations between the parameters of GRM and LKM were used (c.f. [46])

$$
a_{i}=a_{i}^{*}, \quad k_{\mathrm{LKM}, \mathrm{i}}=\left[\frac{a_{i}^{*}}{1-\epsilon}\left(\frac{R_{p}}{3 k_{\mathrm{ext}, i}}+\frac{R_{p}^{2}}{15 D_{\mathrm{eff}, i}}\right)\right]^{-1}, \quad i=1,2 .
$$

However, in the reaction term $a_{i}$ is not replaced by $a_{i}^{*}$. Figure $7(\mathrm{~b})$ verifies that for these values, the variances of GRM and LKM are identical. Moreover, for these values of $a_{i}$ and $k_{\mathrm{LKM}, i}$, the 
concentration profiles of both models are overlapping each other as depicted in Figure 7(c). From the results one concludes that, even a simplified model can give accurate results if all parameters appearing in the model equation are chosen cleverly. Then, simplified models are enough to get physical solutions and there is no need to deal with complicated models.

\subsection{Problem 2: Reversible reaction}

In this section, the semi-analytical and numerical solutions of RGRM are compared with each other for reversible reactions. In the results, only Dirichlet inlet boundary conditions are considered for rectangular pulses injections. Once again, the same values of $D_{\text {eff }, i}$ and $k_{\text {ext }, i}$ are chosen for both components. All model parameters are listed in Table 1.

Figure 8 shows the concentration profiles at the column outlet after injecting a rectangular pulse of finite width in an initially regenerated column $\left(c_{i, \text { init }}=0.0, g / l\right.$ for $\left.i=1,2\right)$ using Dirichlet BCs. Here, the reaction rate constants are taken as $\omega_{1}=0.1$, and $\omega_{2}=0.05$. In Figure 8(a) equal amount of injection is considered for both components $\left(c_{i \text {,inj }}=0.5 \mathrm{~g} / \mathrm{l}\right.$ for $\left.i=1,2\right)$, where as in Figure $8(\mathrm{~b}) c_{\mathrm{inj}, 1}=0.5 \mathrm{~g} / \mathrm{l}$ and $c_{\mathrm{inj}, 2}=0.25 \mathrm{~g} / \mathrm{l}$. Now, a significant impact of reversible reactions is visible, as the concentration levels of both components are different as compared to the concentration levels obtained in the case of Irreversible reactions (c.f. Figure 1). As a result, greater volume of reactant (component 1) is unconverted and lower amount of product (component 2) is produced. However, the conversion rate of reactant into product increases when equal amount of both components is injected to the column. Good agreements of the solution profiles validate the accuracy of numerical Laplace inversion and the HR-FVS.

Finally, the numerical temporal moments of semi-analytical solutions are shown Figure 9 for the case of reversible reaction considering different flow velocities of the mobile phase and Dirichlet BCs. The magnitudes of zeroth moments reflect the obvious effects of flow velocity on the conversion of reactant. The first moments reveal that retention time of component 1 (reactant) is higher than component 2 (product). The plot of second central moments depict that variance (spreading) of both components reduces on increasing the velocity. Further, the third central moments, which quantify the asymmetry of the elution profiles, is also decreasing with increasing the flow rates. 


\section{Conclusion}

Semi analytical solutions of two components linear reactive general rate model were presented considering two sets of boundary conditions. The Laplace transformation was employed to obtain the general analytical solutions in the Laplace domain. The numerical Laplace inversion was used to get the desired concentration profiles in the actual time domain. Moreover, temporal moments were obtained by numerically integrating the semi-analytical solutions. The semi analytical results were validated against the numerical results of a second order finite volume scheme. Good agreements between semi-analytical and numerical results verified the correctness of analytical expressions and accuracy of the suggested numerical scheme. The derived semi-analytical solutions are useful tools to study the influence of reaction rate constants, interfacial mass transfer rate, intra particle pore diffusion, and adsorption affinity on the concentration profiles. These analytical solution can also be used to validate newly introduced numerical schemes.

\section{Appendix}

\section{A. Analytical moments for Dirichlet BCs}

Here, the first two moments are presented Dirichlet boundary conditions. For the derivation of these moments, we have chosen $c_{1 \text {,init }}=0 \mathrm{~g} / \mathrm{l}, c_{2 \text {,init }}=0 \mathrm{~g} / \mathrm{l}$, and $c_{\mathrm{inj}, 2}=0 \mathrm{~g} / \mathrm{l}$. That means, we have considered a regenerated column and injected only component 1 into the reactor. Using Eqs. (107) and (108), we have derived the following moments $\mu_{n}^{(i)}$ of Laplace transformed solutions given by Eqs. (55) and (57) for $i=1,2$ and $n=0,1,2,3$. Let us define

$$
\tilde{r}=\operatorname{PeF} \omega, \quad \gamma=\sqrt{P e^{2}+4 \tilde{r}}, \quad \delta_{1,2}=\operatorname{Pe} \mp \gamma, \quad \tilde{k}_{i}=\left[\frac{a_{i}^{*}}{1-\epsilon}\left(\frac{F}{\xi_{p i}}+\frac{1}{15 \eta_{p i}}\right)\right]^{-1} . \quad i=1,2 .
$$

By using Eq. (107), the zeroth moments are given as

$$
\mu_{0}^{(1)}=c_{\mathrm{inj}, 1} t_{\mathrm{inj}} e^{\frac{\delta_{1}}{2}}, \quad \mu_{0}^{(2)}=c_{\mathrm{inj}, 1} t_{\mathrm{inj}}\left(1-e^{\frac{\delta_{1}}{2}}\right) .
$$

From Eq. (A-2), we get $\mu_{0}^{(1)}+\mu_{0}^{(2)}=c_{1, \text { inj }} \tau_{\text {inj }}$, as $c_{\text {inj }, 2}=0$ is considered. 
The first moments are calculated by using the Eq. (108) for $n=1$

$$
\begin{aligned}
& \mu_{1}^{(1)}=\frac{t_{\text {inj }}}{2}+\frac{L P e\left(1+a_{1}^{*} F\right)}{u \gamma}, \\
& \mu_{1}^{(2)}=\frac{t_{\text {inj }}}{2}+\frac{L}{u} \frac{\operatorname{Pe}\left\{\gamma F\left(a_{2}^{*}-a_{1}^{*}\right)-\tilde{r}\left(1+a_{1}^{*} F\right)\right\} e^{\frac{\delta_{1}}{2}}-\gamma\left\{F\left(a_{2}^{*}-a_{1}^{*}\right) P e-\tilde{r}\left(1+a_{2}^{*} F\right)\right\}}{\tilde{r} \gamma\left(1-e^{\frac{\delta_{1}}{2}}\right)} .
\end{aligned}
$$

The second central moments are expressed as

$$
\mu_{2}^{\prime(i)}=\mu_{2}^{(i)}-\left(\mu_{1}^{(i)}\right)^{2}, \quad i=1,2
$$

Thus, we have

$$
\begin{aligned}
\mu_{2}^{\prime(1)}= & \frac{t_{\text {inj }}^{2}}{12}+\frac{L^{2}}{u^{2}} \frac{2 P e\left[\gamma^{2} a_{1}^{*} F(1-\epsilon)+P e \kappa_{1}\left(1+a_{1}^{*} F\right)^{2}\right]}{\gamma^{3} \kappa_{1}}, \\
\mu_{2}^{\prime(2)}= & \frac{t_{\text {inj }}^{2}}{12}+\frac{L^{2}}{u^{2}} \frac{1}{1-e^{\frac{\delta_{1}}{2}}}\left[e ^ { \frac { \delta _ { 1 } } { 2 } } \left\{-\frac{2 P e a_{1}^{*} F(1-\epsilon)}{\gamma \kappa_{1}}-\frac{2 P e F(1-\epsilon)\left(a_{1}^{*} \kappa_{2}-a_{2}^{*} \kappa_{1}\right)}{\tilde{r} \kappa_{1} \kappa_{2}}-\frac{2 P e^{2}\left(1+a_{1}^{*} F\right)^{2}}{\gamma^{3}}\right.\right. \\
& \left.-\frac{P e^{2} F^{2}\left(a_{2}^{*}-a_{1}^{*}\right)^{2}}{\tilde{r}^{2}}\right\}+\frac{2 P e F(1-\epsilon)\left(a_{1}^{*} \kappa_{2}-a_{2}^{*} \kappa_{1}\right)}{\tilde{r} \kappa_{1} \kappa_{2}}+\frac{P e^{2} F^{2}\left(a_{2}^{*}-a_{1}^{*}\right)^{2}}{\tilde{r}^{2}}+\frac{2\left(1+a_{2}^{*} F\right)^{2}}{P e} \\
& \left.+\frac{2 a_{2}^{*} F(1-\epsilon)}{\kappa_{2}}\right]-\frac{L^{2}}{u^{2}}\left[\frac{P e^{2}\left\{\gamma F\left(a_{2}^{*}-a_{1}^{*}\right)-\tilde{r}\left(1+a_{1}^{*} F\right)\right\}^{2} e^{\frac{\delta_{1}}{2}}-\gamma^{2}\left\{F\left(a_{2}^{*}-a_{1}^{*}\right) P e-\tilde{r}\left(1+a_{2}^{*} F\right)\right\}^{2}}{\tilde{r}^{2} \gamma^{2}\left(1-e^{\frac{\delta_{1}}{2}}\right)}\right] \\
& -\frac{L^{2}}{u^{2}}\left[\frac{P e\left\{\gamma F\left(a_{2}^{*}-a_{1}^{*}\right)-\tilde{r}\left(1+a_{1}^{*} F\right)\right\} e^{\frac{\delta_{1}}{2}}-\gamma\left\{F\left(a_{2}^{*}-a_{1}^{*}\right) P e-\tilde{r}\left(1+a_{2}^{*} F\right)\right\}}{\tilde{r} \gamma\left(1-e^{\frac{\delta_{1}}{2}}\right)}\right]^{2}
\end{aligned}
$$

\section{References}

[1] Ruthven, D.M., 1984. Principles of adsorption and adsorption processes. Wiley-Interscience, New York.

[2] Ganetsos, G., Barker, P.E., 1993. Preparative and production scale chromatography (vol. 61). Marcel Dekker, Inc., New York, 375-523.

[3] Guiochon, G., Felinger, A., Shirazi, D.G., Katti, A.M., 2006. Fundamentals of preparative and nonlinear chromatography, 2nd ed. ELsevier Academic press, New York. 
[4] Lin, B., Song, F., Guiochon, G., 2003. A nalytical solution of the ideal, nonlinear model of reaction chromatography for a reaction $\mathrm{A} \leftarrow \mathrm{B}$ and a parabolic isotherm. J. Chromatogr. A. 1003, 91-100.

[5] Carta, G., 1991. Simultaneous reaction and chromatography, in Chromatographic and Membrane Processes in Biotechnology (Edited by C. A. Costa and J. S. Cabral), 429-447, NATO ASI, Kluwer Academic Publishers, Deventer.

[6] Schweich, D., Villermaux, J., 1978. The chromatographic reactor. A new theoretical approach. Ind. Eng. Chem. Fundamen, 17, 1-7.

[7] Cho, B.K., Aris, R., Carr, R.W., 1982. The mathematical theory of a countercurrent catalytic reactor. Proc. R. Sot. Lond. 383, 147-189.

[8] Petroulas, T., Aris, R., Carr, R.W., 1985. Analysis of the counter-current moving-bed chromatographic reactor, Comp. Math. Appl. 11, 5-34.

[9] Takeuchi, K., Uraguchi, Y., 1976. Separation conditions of the reactant and the product with a chromatographic moving bed reactor. J. Chem. Eng. Japan 9, 164-166.

[10] Takeuchi, K., Uraguchi, Y., 1976. Basic design of chromatographic moving-bed reactors for product refining. J. Chem. Engng Japan 9, 246-248.

[11] Takeuchi, K., Uraguchi, Y., 1977. The effect of the exhausting section on the performance of a chromatographic moving-bed reactor. J. Chem. Engng Japan 10, 72-74.

[12] Takeuchi, K., Uraguchi, Y., 1977. Experimental studies of a chromatographic moving-bed reactor-catalytic oxidation of carbon monoxide on activated alumina as a model reactor, J. Chem. Eng. Japan 10, 455-460.

[13] Takeuchi, K., Miyauchi, T., Uraguchi, Y., 1978. Computational studies of a chromatographic moving bed reactor for consecutive and reversible reactions. J. Chem. Eng. Japan 11, 216-220.

[14] Binous, H., McCoy,B., 1992. Chromatographic reactions of the three components: Application to separations. Chem. Eng. Sci. 47, 4333-4343. 
[15] Borren, T., Fricke, J., Schmidt-Traub, H. (Eds.), 2005. Chromatographic Reactors in Preparative Chromatography of Fine Chemicals and Pharmaceutical Agents. Wiley-VCH Verlag, Weinheim, 371-395.

[16] Fricke, J., Schmidt-Traub, H., Kawase, M., 2005. Chromatographic reactor. Ullmann's Encyclopedia of Industrial Chemistry. Wiley-VCH Verlag, Weinheim.

[17] Javeed, S., Qamar, S., Seidel-Morgenstern, A., Warnecke, G., 2011. A discontinuous Galerkin method to solve chromatographic models. J. Chromatogr. A. 1218, 7137-7146.

[18] Javeed, S., Qamar, S., Seidel-Morgenstern, A., Warnecke, G., 2012. Parametric study of thermal effects in reactive liquid chromatography. Chem. Eng. J. 191, 426-440.

[19] Qamar, S., Bibi, S., Khan, F.U., Shah, M., Javeed, s., Seidel-Morgenstern, A., 2014. Irreversible and reversible reactions in a liquid chromatographic column: Analytical solutions and moment analysis. I\&EC research. 53, 2461-2472.

[20] Bibi, S., Qamar, S., Seidel-Morgenstern, A., 2015. Irreversible and reversible reactive chromatography: Analytical solutions and moment analysis for rectangular pulse injections. J. Chromatogr. A. 1385, 49-62.

[21] Yamaoka, K., Nakagawa, T., 1976. Moment analysis for reaction chromatography. J. Chromatogr. A. 117, 1-10.

[22] Sardin, M., Schweich, D; Villermaux, J., Ganetsos, G., Barker, P.E. (Eds.), 1993. Preparative fixed-bed chromatographic reactor, preparative and production scale chromatography. Marcel Dekker Inc., New York, USA, 477.

[23] Villermaux, J. in: A.E. Rodrigues, D. Tondeur (Eds.), 1981. The Chromatographic Reactor in Percolation Processes: Theory and Application. Sijthoffen Noordhoff, Alpena an den Rijn, The Netherlands, 539-588.

[24] Rodrigues, A., Tondeur, D., 1973. Modéles mathématiques des colonnes échaneuses d'ionsune revue critique: I- Modéles type cinétique chimique, Rev. Port. Quim. 15, 141-157. 
[25] Rodrigues, A., Tondeur, D., 1975. Influence of axial dispersion on chemical conversion. Application to reactor design, Rev. Port. Quim. 17, 183-190.

[26] van der Laan, G.p., , Beenackers, A.A.C.M., Krishna, R., 1999. Multicomponent reaction engineering model for Fe-catalyzed FischerTropsch synthesis in commercial scale slurry bubble column reactors. Chem. Eng. Sci. 54, 5013-5019.

[27] Rosen, J. B., Warren, E. Winsche., 1950. The admittance concept in the kinetics of chromatography. J. Chem. Phys. 18, 1587-1592.

[28] Rosen, J. B., 1952. Kinetics of a fixed bed system for solid diffusion into spherical particles. J. Chem. Phys. 20(3), 387-394.

[29] Rosen J. B., 1954. General numerical solution for solid diffusion in fixed beds. Ind. Eng. Chem. 46(8), 1590-1594.

[30] Massaldi. H. A., Gottifredi, J. C., 1972. Adsorption dans un lit fixe-cas de trois résistances simultanées. Chem. Eng. Sci. , 27, 1951-1956.

[31] Li, P., Xiu, G., Rodrigues, A.E., 2003. Analytical solutions for breakthrough curves in a fixed bed of shell-core adsorbent, AIChE J. 49, 2974-2979.

[32] Li, P., Xiu, G., Rodrigues, A.E., 2003. Modeling breakthrough and elution curves in a fixed bed of shell-core adsorbent for separation of proteins-exact analytical solution and approximate solutions. Chem. Eng. Sci. 59, 3091-3103.

[33] Li, P., Yu, J., Xiu, G., Rodrigues, A.E., 2011. Perturbation chromatography with inert core adsorbent: Moment solution for two-component nonlinear adsorption isotherm. Chem. Eng. Sci. $66,4555-4560$.

[34] Javeed, S., Qamar, S., Ashraf, W., Seidel-Morgenstern, A., Warnecke, G., 2013. Analysis and numerical investigation of two dynamic models for liquid chromatography. Chem. Eng. Sci. $90,17-31$. 
[35] Qamar, S., Abbasi, J., Mehwish, A., Seidel-Morgenstern, A., 2015. Linear general rate model of chromatography for coreshell particles: Analytical solutions and moment analysis. Chem. Eng. Sci. 137, 352-363.

[36] Leweke, S., von Lieres, E., 2016. Fast arbitrary order moments and arbitrary precision solution of the general rate model of column liquid chromatography with linear isotherm. J. Comput. Chem. Eng. 84, 350-362.

[37] Schneider, P., Smith, J.M., 1968. Adsorption rate constants from chromatography, AIChE J. 14, 762-771.

[38] Mehta, R.V., Merson, R.L., McCoy, B.J., 1974. Hermite polynomial representation of chromatography elution curves, J. Chromatography A 88, 1-6.

[39] Koren, B., 1993. A robust upwind discretization method for advection, diffusion and source terms. In C. B. Vreugdenhil, B. Koren, editors, Numerical Methods for Advection-Diffusion Problems, Volume 45 of Notes on Numerical Fluid Mechanics, chapter 5, pages 117-138, Vieweg Verlag, Braunschweig..

[40] Javeed, S., Qamar, S., Seidel-Morgenstern, A., Warnecke, G., 2011. Efficient and accurate numerical simulation of nonlinear chromatographic processes. J. Comput. Chem. Eng. 35, 22942305.

[41] Qamar, S., Sattar, F.A., Abbasi, J., Seidel-Morgenstern, A., 2016. Numerical simulation of nonlinear chromatography with core-shell particles applying the general rate model. Chem. Eng. Sci. 147, 54-64.

[42] Danckwerts, P.V., 1953. Continuous flow systems. Chem. Eng. Sci. 2, 1-9.

[43] Quezada, C.R., Clement, T.P., Lee, K.K., 2004. Generalized solution to multi-dimensional multi-species transport equations coupled with a first-order reaction network involving distinct retardation factors. Advances in water resources 27, 507-520.

[44] Rice, R.G., Do, D.D., 1995. Applied mathematics and modeling for chemical engineers. Wiley-Interscience, New York. 
[45] Durbin, F., 1974. Numerical Inversion of Laplace Transforms: An efficient improvement to Dubner and Abate's Method. The Computer J. 17, 371-376.

[46] Qamar, S., Abbasi, J., Javeed, S., Seidel-Morgenstern, A., 2014. Analytical solutions and moment analysis of general rate model of linear reactive chromatography. Chem. Eng. Sci. 107, 192-205. 
Table 1: Parameters of linear reactive two-component GRM.

\begin{tabular}{|c|c|}
\hline Parameters & values \\
\hline Column length & $L=10 \mathrm{~cm}$ \\
Interstitial velocity & $u=2.5 \mathrm{~cm} / \mathrm{min}$ \\
Axial dispersion coefficient & $D_{z}=0.34 \mathrm{~cm}^{2} / \mathrm{min}$ \\
Effective dispersion coefficient & $D_{\text {eff }}=10^{-4} \mathrm{~cm}^{2} / \mathrm{min}$ \\
External mass transfer coefficient & $k_{\text {ext }}=0.01 \mathrm{~cm} / \mathrm{min}$ \\
External porosity & $\epsilon=0.4$ \\
Internal porosity & $\epsilon_{p}=0.333$ \\
Total simulation time & $t_{\mathrm{max}}=50 \mathrm{~min}$ \\
Initial concentrations & $c_{\mathrm{init}, i}=0 \mathrm{~g} / \mathrm{l}$ \\
Concentration at inlet for component 1 & $c_{\mathrm{inj}, 1}=0.5 \mathrm{~g} / \mathrm{l}$ \\
Concentration at inlet for component 2 & $c_{\mathrm{inj}, 2}=0.5 \mathrm{~g} / \mathrm{l}$ \\
Adsorption equilibrium constant for component 1 & $a_{1}=2.5$ \\
Adsorption equilibrium constant for component 2 & $a_{2}=0.5$ \\
Reaction rate constant (component 1) & $\omega_{1}=0.1$ \\
Reaction rate constant (component 2) & $\omega_{2}=0.05$ \\
\hline
\end{tabular}



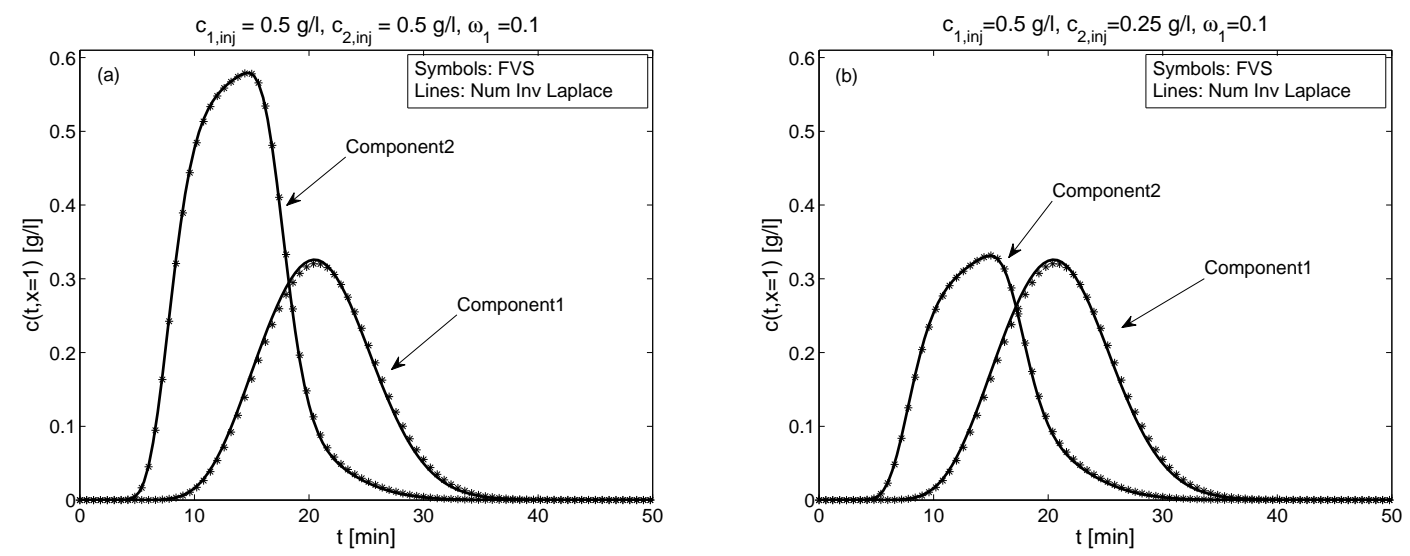

Figure 1: Irreversible reaction: Effects of injection on the concentration profiles obtained by Dirichlet BCs at $x=1$.
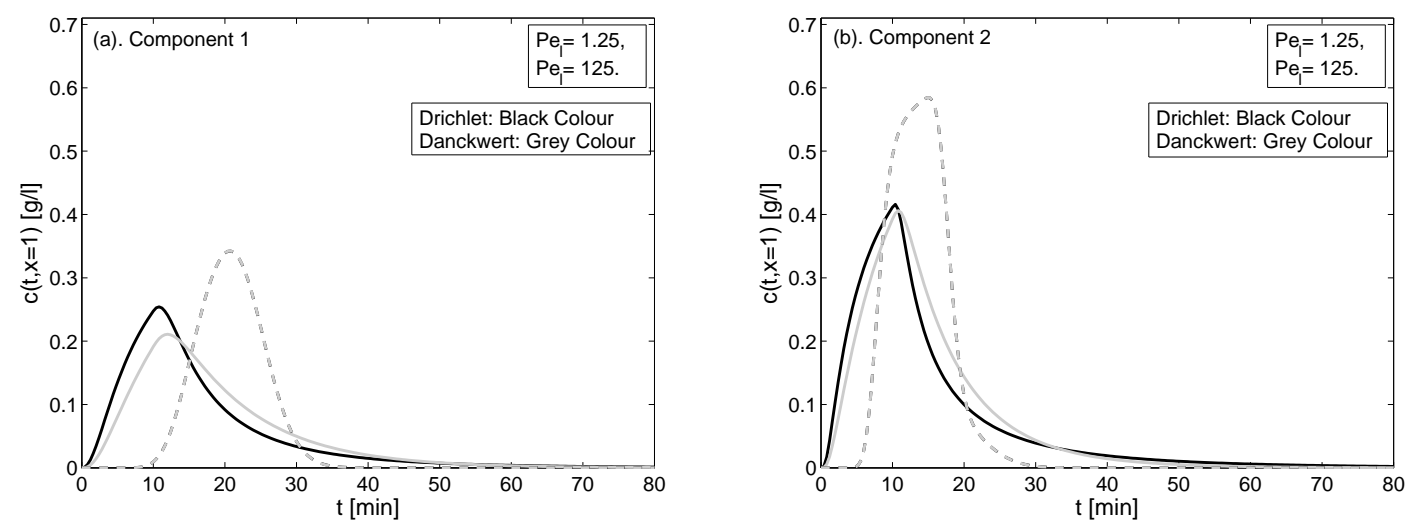

Figure 2: Irreversible reaction: Effects of Dirichlet and Danckwerts BCs on the concentration profiles at $x=1$. Here, $c_{\mathrm{inj}, 1}=0.5 \mathrm{~g} / \mathrm{l}, c_{\mathrm{inj}, 2}=0.5 \mathrm{~g} / \mathrm{l}$ and $\omega_{1}=0.1$. 


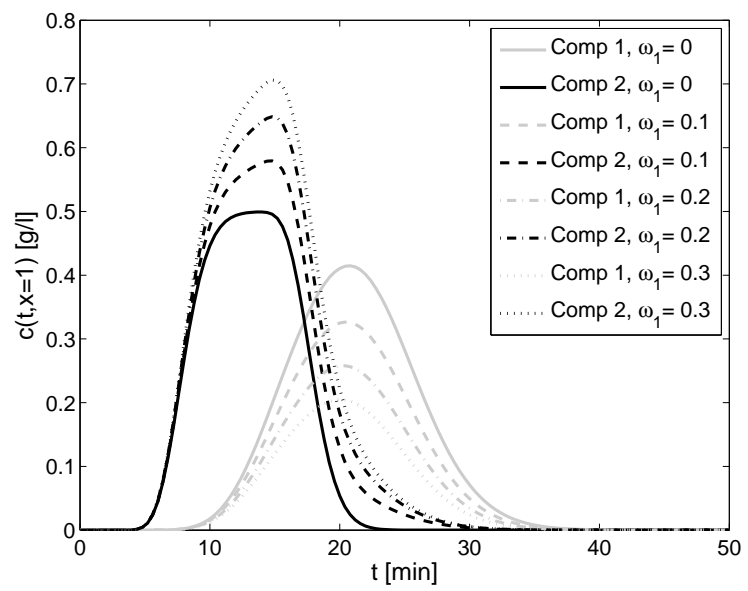

Figure 3: Irreversible reaction: Effects of reaction rate constant $\omega_{1}$ on the concentration profiles at $x=1$, using Danckwerts BCs. Here, $c_{\mathrm{inj}, 1}=0.5 \mathrm{~g} / \mathrm{l}$ and $c_{\mathrm{inj}, 2}=0.5 \mathrm{~g} / \mathrm{l}$.
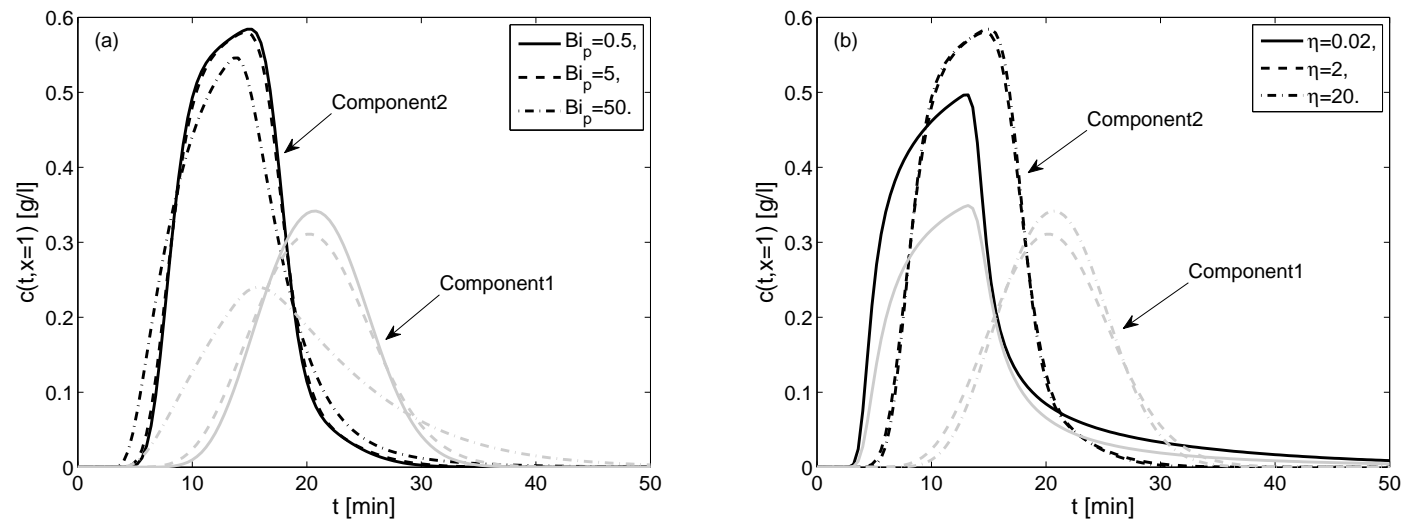

Figure 4: Irreversible reaction: (a): Effects of $B i_{p}$ on the concentration profiles. (b): Effects of $\eta_{p}$ on the concentration profiles. 

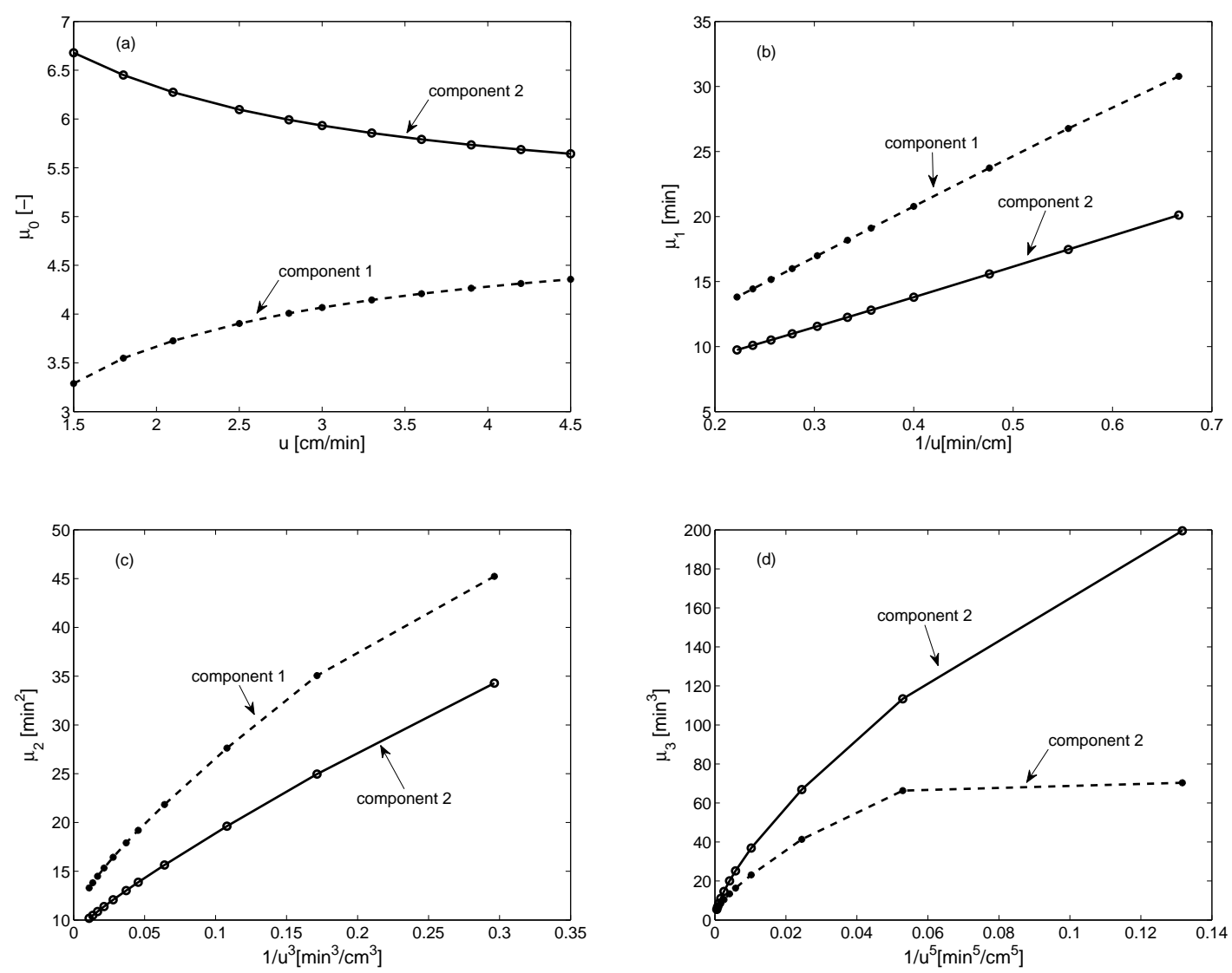

Figure 5: Irreversible reaction: Plots of central moments (numerical) considering different flow rates using Dirichlet BCs. Here, $c_{\mathrm{inj}, 1}=0.5 \mathrm{~g} / \mathrm{l}, c_{\mathrm{inj}, 2}=0.5 \mathrm{~g} / \mathrm{l}, \omega_{1}=0.1$. 

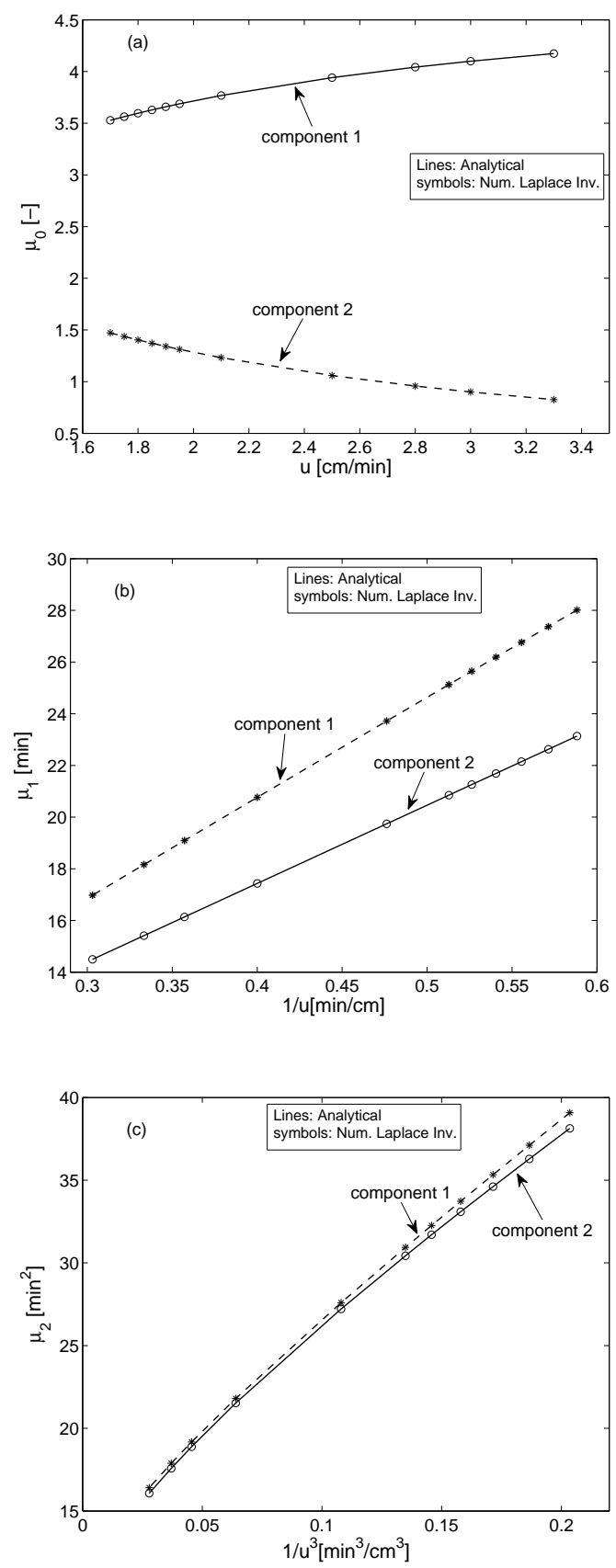

Figure 6: Irreversible reaction: A comparison of analytical central moments (Eqs. (A-2)-(A-7)) and numerical moments of Laplace inversion solution for different flow rates using Dirichlet BCs, $c_{\mathrm{inj}, 1}=0.5$, $c_{\mathrm{inj}, 2}=0$, and $\omega_{1}=0.1$. 

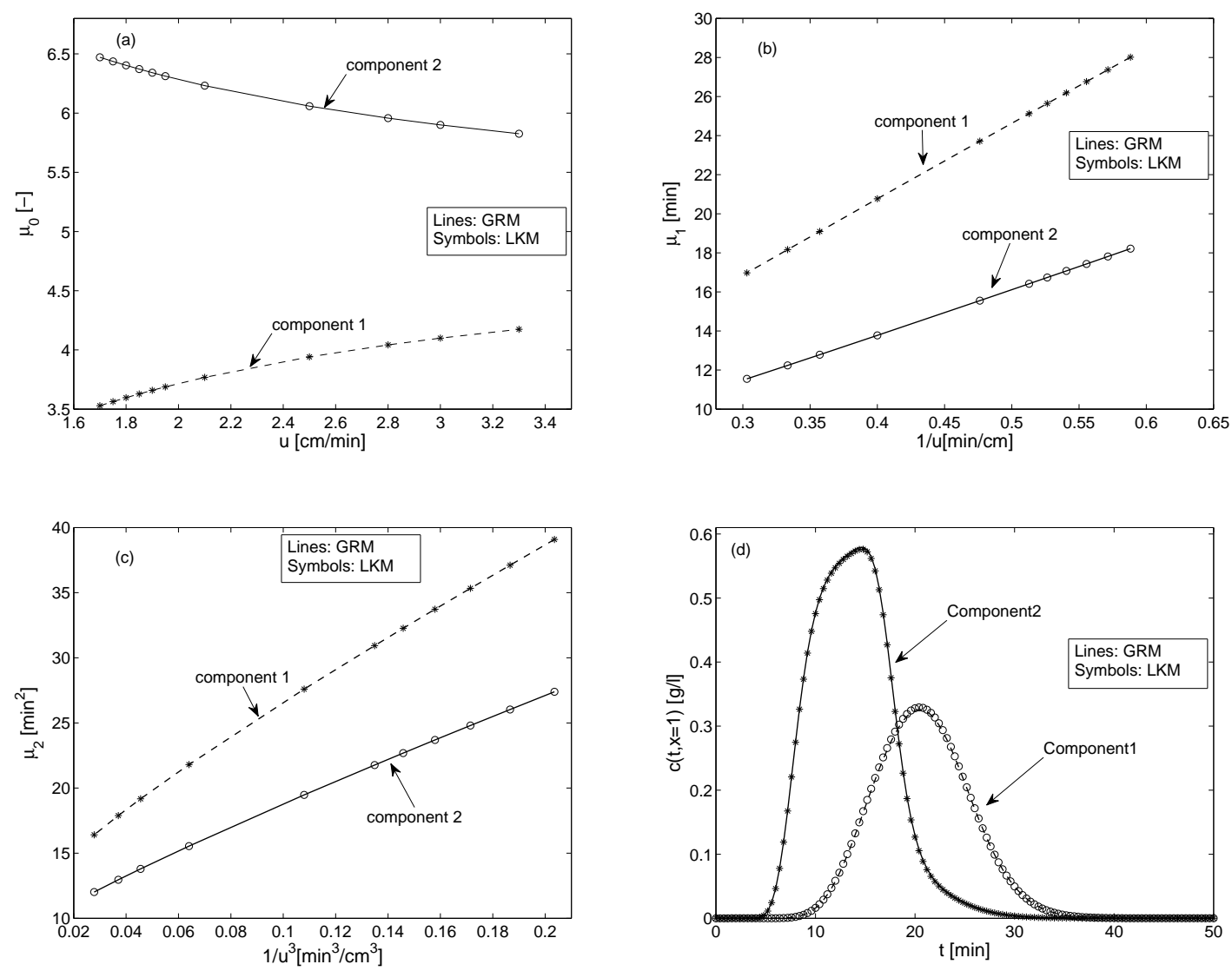

Figure 7: Irreversible reaction: Moments and concentration profiles of LKM and GRM when parameters of both models are matched through Eqs. (111) and (112). Here $\omega=0.1, u=2.5 \mathrm{~cm} / \mathrm{min}$ and other parameters are given in Table 1. 

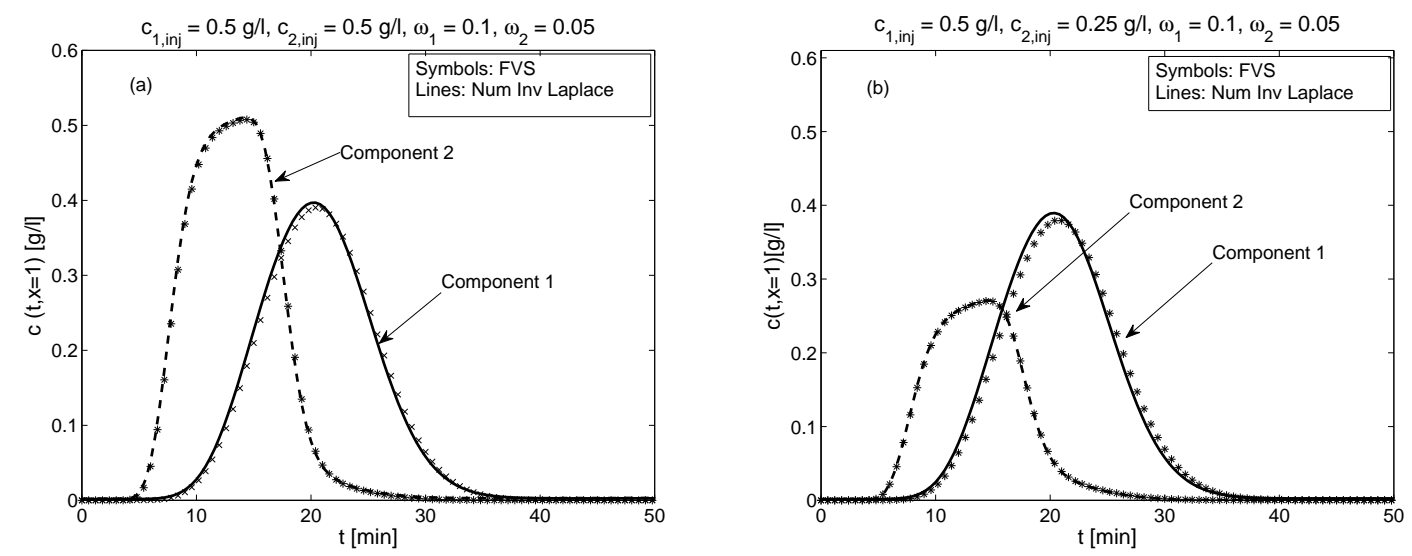

Figure 8: Reversible reaction: Effects of injection on the concentration profiles at $x=1$. All parameters are given in Table 1 .
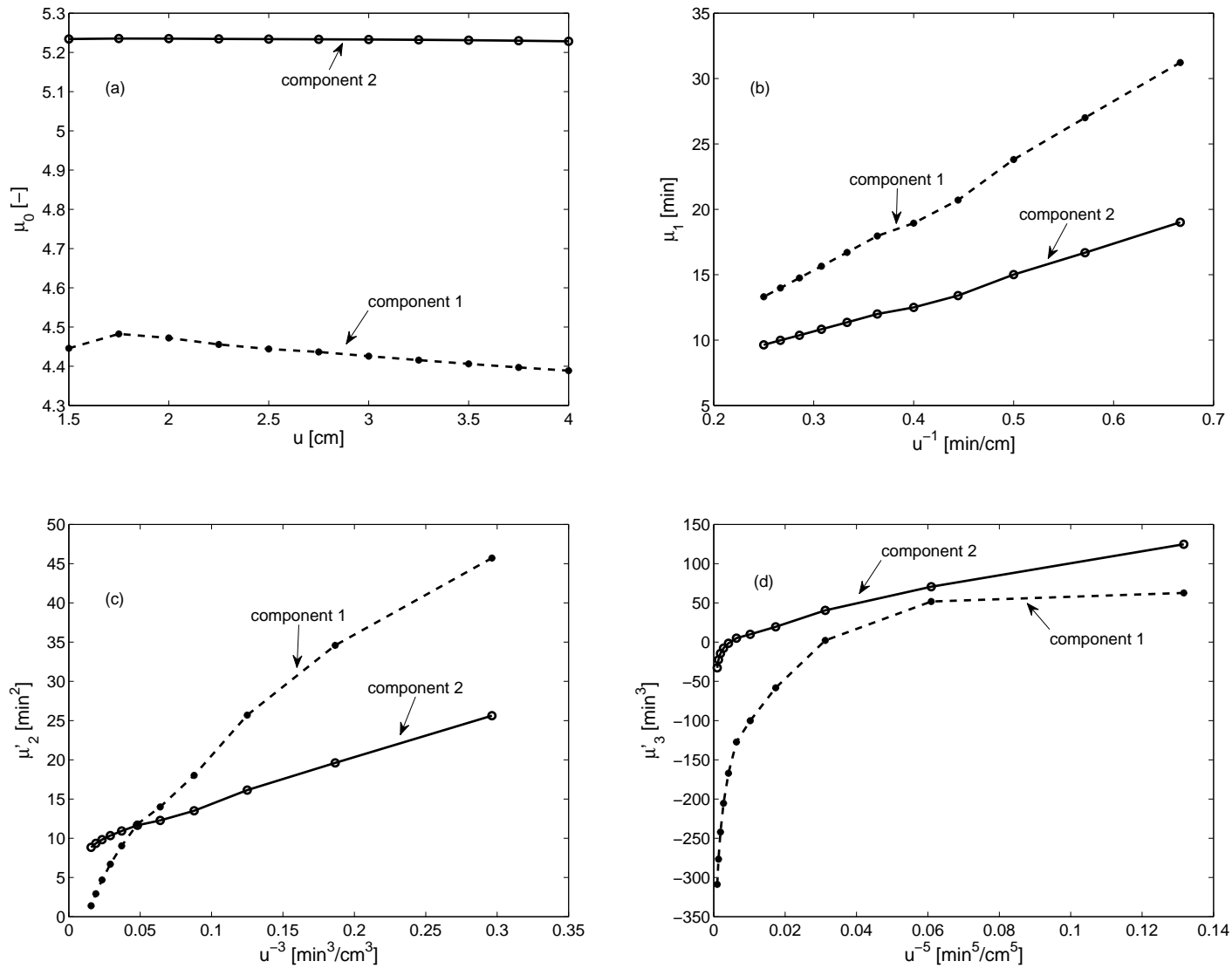

Figure 9: Reversible reaction: Plots of central moments (numerical) considering different flow rates using Dirichlet BCs. Here, $c_{\mathrm{inj}, 1}=0.5 \mathrm{~g} / \mathrm{l}, c_{\mathrm{inj}, 2}=0.5 \mathrm{~g} / \mathrm{l}, \omega_{2}=0.1$ and $\omega_{2}=0.05$. 